\title{
Passive flow control study in an S-duct intake using Stereo Particle Image Velocimetry
}

\author{
Geoffrey Tanguy ${ }^{1}$, David G. MacManus ${ }^{2}$ (MAIAA), Pavlos Zachos ${ }^{3}$, Daniel Gil-Prieto ${ }^{4}$ \\ Propulsion Engineering Centre, School of Aerospace, Transport and Manufacturing, Cranfield University, MK43 \\ $O A L, U K$ \\ Eric Garnier ${ }^{5}$ \\ ONERA, The French Aerospace Lab, Meudon, 92190, France
}

The ability of vortex generators (VG) to reduce the unsteady distortion at the exit Aerodynamic Interface Plane (AIP) of an S-duct is investigated. The 3 components of the velocity at the AIP were measured using a Stereo Particle Velocimetry system with high spatial resolution. This enabled an assessment of the synchronous swirl distortion at the duct exit. A total of nine VG cases have been investigated with a systematic variation of key design variables. Overall the VGs change the duct secondary flows and separation and are able to substantially restructure the flow field at the AIP. The pressure distortion could be reduced up to $50 \%$ and a reduction in pressure loss of $30 \%$ was achieved for the mean flow field. The VGs have a substantial influence on the unsteadiness of the flow field with a reduction in peak swirl unsteadiness of $61 \%$ and an overall reduction of unsteady swirl distortion of $67 \%$. They also suppress the primary unsteady flow switching mechanism of the datum configuration which is associated with the oscillation of bulk and twin swirl regimes. Consequently, extreme events which leads to high swirl intensity are suppressed which lower by $45 \%$ the maximum swirl intensity for the VG cases.

$A \quad=$ S-duct cross section area, $\mathrm{m}^{2}$

\section{Nomenclature}

$c=$ Vortex generator length, $\mathrm{mm}$

$D \quad=\quad$ S-duct cross section diameter, $\mathrm{mm}$

${ }^{1}$ PhD Student, Propulsion Engineering Centre, Building 52, Cranfield University

${ }^{2}$ Senior Lecturer, Propulsion Engineering Centre, Building 52, Cranfield University, AIAA Member.

${ }^{3}$ Lecturer, Propulsion Engineering Centre, Building 52, Cranfield University, AIAA Member.

${ }^{4} \mathrm{PhD}$ Student, Propulsion Engineering Centre, Building 52, Cranfield University.

${ }^{5}$ Research Scientist, Applied Aerodynamics Department, 8 Rue des Vertugadins 


$$
\begin{array}{ll}
D C 60 & =60^{\circ} \text { sector distortion coefficient } \\
H & =\text { S-duct centerline offset, } \mathrm{mm} \\
h & =\text { Vortex generator height, } \mathrm{mm} \\
L & =\text { S-duct axial length, } \mathrm{mm} \\
l s & =\text { Vortex generator lateral spacing, } \mathrm{mm} \\
M & =\text { Mach number } \\
P_{0} & =\text { Total pressure, Pa } \\
P R & =\text { Pressure recovery ratio } \\
q & =\text { Mean dynamic head, Pa } \\
R & =\text { S-duct cross section radius, mm } \\
r & =\text { Radial coordinate from the AIP center, mm } \\
\mathrm{Re} & =\text { Reynolds number based on the inlet diameter } \\
\mathrm{SI} & =\text { Swirl Intensity distortion descriptor, deg } \\
\mathrm{SP} & =\text { Swirl Pairs distortion descriptor } \\
\mathrm{SD} & =\text { Swirl Directivity distortion descriptor } \\
u, v, w & =\text { Cartesian velocity vector components, } \mathrm{m} / \mathrm{s}
\end{array}
$$

\section{Greek symbols}

$$
\begin{array}{ll}
\alpha & =\text { Swirl angle, deg } \\
\alpha_{\mathrm{s}} & =\text { Vortex generator angular spacing, deg } \\
\beta_{\mathrm{s}} & =\text { Vortex generator toe angle, deg } \\
\Delta x & =\text { Distance between VG centerline axial position and nominal separation point, mm } \\
\Delta \eta & =\text { Relative loss in total pressure ratio } \\
\delta & =\text { Boundary layer thickness, mm } \\
\theta_{s} & =\text { Circumferential extent in which the vortex generator are placed, deg }
\end{array}
$$

\section{Operators}

$$
\begin{array}{ll}
\langle\cdot \quad & =\text { time average } \\
\cdot & =\text { area weighted average } \\
\sigma & =\text { standard deviation }
\end{array}
$$




\section{Subscripts}

$$
\begin{array}{ll}
\text { AIP } & =\text { Aerodynamic Interface Plane }\left(0.24 \mathrm{D}_{\text {out }} \text { downstream the S-duct outlet plane }\right) \\
\text { in } & =\text { S-duct inlet plane } \\
\text { out } & =\text { S-duct outlet plane } \\
\text { ref } & =\text { Reference plane }\left(0.9 \mathrm{D}_{\text {in }} \text { upstream the S-duct inlet plane }\right)
\end{array}
$$

\section{Introduction}

$\mathrm{E}$ complex aero-engine intakes. These types of intakes are expected to play a major role in the next generation of aircraft as part of an alternative to conventional transport configurations [1]. Fully or partially integrated propulsion systems are used for their potential overall benefit with possible reductions in drag and noise. However, S-shaped intake diffusers for the propulsion system are susceptible to high levels of flow unsteadiness and distortion. This is typically driven by secondary flows and local flow separations [2,3]. As a result, complex total pressure and swirl distortion fields, with significant vortical regions and large unsteady perturbations, are presented to the first stage of the compression system. This can adversely affect the whole engine performance, operability and structural integrity $[4,5]$.

The potentially negative impact of distortion on the turbomachinery components for embedded propulsion system has received notable attention [5-10]. However, it is still one of the main challenges regarding the design of complex intakes. The effect of steady bulk swirl on the compression system is relatively well understood with a change of blade loading which affects the surge margin. Previous studies have shown that the introduction of steady counter-rotating swirl with total pressure distortion at the inlet of a compressor can significantly reduce, or erase, the stability margin [4]. In addition, flow distortions associated with an S-duct configuration are unsteady which can also promote stall inception in the compressor system [7,10]. 


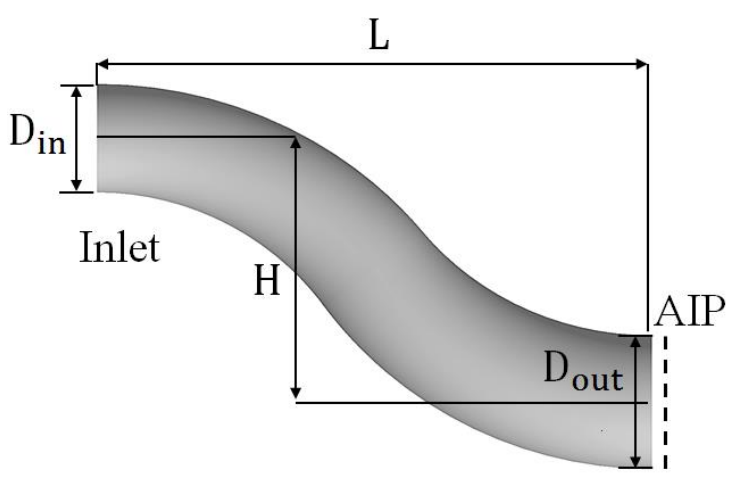

Outlet

Fig. 1 S-duct geometry definition

Previous studies based on time averaged flow field measurements or steady computational fluid dynamics (CFD) simulations, highlighted a pair of counter-rotating vortices at the Aerodynamic Interface Plane (AIP) at the duct exit. This is associated with a loss in total pressure in the lower sector of the AIP [3,11,12]. However, it was demonstrated by Berens et al. [13] that time averaged data can be misleading, and the use of Delayed Detached Eddy Simulations (DDES) revealed the notable flow unsteadiness present at the AIP for an S-duct (Fig. 1) $\left(\mathrm{A}_{\text {out }} / \mathrm{A}_{\text {in }}\right.$ $\left.=1.4, \mathrm{~L} / \mathrm{D}_{\text {in }}=4.67, \mathrm{H} / \mathrm{L}=0.23\right)$. It was also concluded that the assessment of distortion has to take into account the unsteadiness of the flow field. Garnier [14] investigated the AIP flow field for an S-duct $\left(\mathrm{A}_{\text {out }} / \mathrm{A}_{\text {in }}=1.52, \mathrm{~L} / \mathrm{D}_{\text {in }}=4.95\right.$, $\mathrm{H} / \mathrm{L}=0.50$ ) based on unsteady total pressure data acquired with 40 high-bandwidth transducers. High levels of total pressure fluctuations were identified at the center of the AIP with the postulation of a lateral oscillation of the loss region associated with the main secondary flow vortices. Zachos et al. [15] applied Stereoscopic Particle Image Velocimetry (S-PIV) to measure the distorted velocity field at the outlet of two S-duct configurations with different vertical offsets $(H / L=0.27,0.50)$. The synchronous, high spatial resolution measurements revealed the unsteady nature of the flow field for an S-duct which is the same geometry as that studied in this paper. Strong streamwise velocity fluctuations were identified close to the center of the AIP with regions of maximum fluctuation postulated to be due to shear layer oscillations associated with the centerline separation. The swirl angle fluctuations at the AIP were found to be driven by the unsteady circumferential velocity linked to the strong secondary flows which arise within curved ducts. Furthermore, notable excursions from the mean flow field was observed and characterized by the flow distortion descriptors. The assessment of the flow field based on each snapshot revealed the presence of bulk swirling structures rotating either clockwise or anti-clockwise. Gil-Prieto et al. [16] identified the inner ring $(\mathrm{r} / \mathrm{R}=0.32)$ of the AIP section as the most critical in terms of unsteady swirl distortion for the same duct. The 
analysis pointed out a tri-modal state of oscillation of the flow corresponding to a positive bulk swirl, twin swirl and negative bulk swirl. Peak values of swirl intensity in the inner rings were associated with the bulk swirl pattern. GilPrieto et al. [16] also applied proper orthogonal decomposition (POD) on the velocity vector field at the AIP to identify the most energetic coherent structures of the flow field. This highlighted two main mechanisms present in the distorted flow field; a swirl switching mode and a vertical-perturbation mode [16].

Historically, distortion at the AIP for convoluted intakes has been partially addressed using flow control methods which influence the flow structure inside the diffuser and prevent separation region. A number of previous studies have investigated the use of passive flow control to reduce the steady distortion at the AIP for both rectangular [1719] and circular ducts [11,20-23]. Generally, in the form of arrays of co-rotating vortex generators (VGs), passive flow control devices are used to modify the secondary flows inside the duct.

In addition, active flow control methods such as pulsed or continuous air jets [14,24] have also been studied. Garnier [14] investigated the use of active flow control to successfully suppress the flow separation of an S-duct which is the same as that investigated in the current work. The analysis revealed the sensitivity of the distortion at the AIP to the jet frequency. For continuous blowing jets, the DC60 was improved from 0.25 to 0.12 at an AIP Mach number of 0.4. An increase in unsteady distortion was observed when the pulsed jets were used. The combination of both passive and active flow control devices were also investigated to reduce the risk of failure by only using active control [18,25-27]. In addition to providing a fail-safe mechanism with added adjustable control over the flow, an overall reduction of $35 \%$ of the distortion was achieved by the use of the hybrid control system compared with the case without flow controls [25].

The primary mechanism of vortex generators is to re-energize the boundary layer at the pre-separation stage in order to increase the aerodynamic performance. Vortex generators can be used to effectively supress local separation regions that occur in S-ducts and improve the distortion levels at the AIP. However the experimental research of Reichert and Wendt [11] demonstrated that this mixing enhancement of the boundary layer is not sufficient to effectively reduce the distortion in complex intakes. As part of these previous studies, thin tapered vortex generators located upstream of the separation point, in a co-rotating pattern oriented outward from the centerline of duct were used. This system redirected the flow and reduced the migration of the azimuthal secondary flow and subsequently impacted onto the distortion at the AIP. Reichert and Wendt [11] concluded that the capacity of the VGs to also turn the local flow field and to suppress the natural secondary flows was partially responsible for the improvement in the 
aerodynamic performance. The investigation comprised a variation in the numbers of VG and lateral spacing (ls) with a VG height in the same order or magnitude as the local boundary layer thickness $\left(\mathrm{h} / \delta_{\mathrm{in}}=1.35\right)$. An overall improvement in pressure based distortion descriptors at the AIP was achieved with a reduction of up to about 50\% in DC60. The different configurations had a notable impact on the flow field and resulted in a wide range of flow topologies at the AIP. For example, closely spaced VG configurations $\left(1 \mathrm{~s} / \mathrm{D}_{\mathrm{in}}=0.156\right)$ generated a single pair of contra rotating vortices at the AIP. The configuration with relatively large spacing $\left(1 \mathrm{~s} / \mathrm{D}_{\mathrm{in}}=0.5\right)$ indicated the presence of independent multiple pairs of vortices at the AIP. Both sets of configurations led to a rise in static pressure at the duct exit and an increase in the total pressure recovery (PR) of the diffuser between $0.40 \%$ and $0.78 \%$. The measurements also demonstrated that for widely spaced VGs $\left(1 \mathrm{~s} / \mathrm{D}_{\mathrm{in}}=0.5\right)$, a global improvement of the flow field at the AIP could be achieved without systematically suppressing separation.

Anderson and Gibb [21] investigated the effect of rectangular VGs to reduce the distortion at the AIP on an Sduct geometry $\left(\mathrm{A}_{\text {out }} / \mathrm{A}_{\text {in }}=1.4, \mathrm{H} / \mathrm{L}=0.3\right)$. A reduction in DC60 of up to $80 \%$ at the AIP was achieved with a VG height $h=0.58 \delta_{\text {in }}$, a VG centerline axial position at $2 \mathrm{R}_{\text {in }}$ from the inlet and a number of VGs of 22 covering a circumferential sector of $157.5^{\circ}$. The comparison of the numerical and experimental data [22] showed the corotating VG configuration was used to redistribute the low energy flow uniformly around the periphery of the engine face leaving a high energy central region.

Recent investigations used the combination of RANS calculation and optimization algorithms to find the optimum VG configuration in terms of distortion reduction at the AIP [23,28,29]. Yi et al. [28] performed an optimization for an S-duct geometry $\left(\mathrm{A}_{\text {out }} / \mathrm{A}_{\text {in }}=1.4, \mathrm{H} / \mathrm{L}=0.3\right)$ using two different reduced-order computational models based on a VG configuration developed by Anderson and Gibbs [21]. These results showed that the DC60 could be reduced by up to $96 \%$ while maintaining the pressure recovery ratio. Jirasek $[29,30]$ performed several optimization studies using passive flow control devices with a vortex generator model implemented in a CFD method. It was found that the VG height and distance from the initial start of the separation were the two most important parameters. The CFD results predicted a decrease in DC60 up to $72 \%$ for an improvement of total pressure recovery of $1.5 \%$ in the case of a double curvature serpentine duct [31] with the combination of VG rows placed at two different locations. The equivalent experimental measurements showed a reduction of DC60 of 59\% [31]. 
Delot et al. [32] measured the total pressure at the AIP with 40 high frequency pressure probes as well as the static pressure along a reduced scale version of the S-duct $\left(\mathrm{A}_{\text {out }} / \mathrm{A}_{\text {in }}=1.52, \mathrm{H} / \mathrm{D}_{\text {in }}=1.34, \mathrm{H} / \mathrm{L}=0.27\right)$ previously investigated by Wellborn et al. [3]. Several sets of vortex generator were investigated. The DC60 was reduced by up to $56 \%$ with a decrease in total pressure peak fluctuations of $33 \%$. This test case was used by the $2^{\text {nd }}$ AIAA Propulsion Aerodynamic Workshop where Scharnhorst and Delot [33] compiled the results of 53 simulations which were compared with the steady experimental data. Overall, the pressure recovery and distortion levels at the AIP could not be matched simultaneously by the computational methods which were also sensitive to the turbulence model.

Although there have been previous studies to investigate the effect of vortex generators on the intake flow distortion, most of the experimental and CFD investigations are based on time averaged data and only a few reported unsteady results. For example, Anderson and Gibb [21] demonstrated a reduction of the unsteady total pressure levels at the AIP by using vortex generators. The results also demonstrated a non-linear increase of the total pressure fluctuations with the Mach number. Delot et al. [32] measured a reduction of 30\% in the unsteady total pressure due to the vortex generators. A spectral analysis of the pressure fluctuations at the AIP showed that VGs effectively reduced the energy content of the frequencies associated with the largest fluctuations for the baseline configuration. However, these previous studies had relatively limited spatial resolution provided by a conventional 40 probe rake and there was no information on the unsteady flow angularity or velocity field at the AIP. The characterization of the swirl distortion ahead of the compressor is one of the major challenges as it directly influences the operability of the engine [4]. However, there is no previous work which investigates the effect of vortex generators on the unsteady swirl distortion. Therefore, the focus of this research is to use high-spatial resolution synchronous S-PIV measurements to investigate the effect of a range of vortex generator configurations on the unsteady velocity and dynamic swirl flow field.

The aim of the present work is to assess the effect of vortex generators on both the steady and for the first time unsteady velocity flow field at the AIP for an S-duct based on high-resolution, synchronous S-PIV measurements. The effect of several VG configurations on the flow field at the AIP is also evaluated in order to provide general design guidelines for future research. The distortion of the mean flow field at the AIP is assessed with conventional pressure and swirl descriptors. However, a statistical approach is used to assess the unsteady swirl distortion at the AIP obtained through synchronous S-PIV measurements. 


\section{Methodology}

\section{A. Experimental facility}

The experimental investigation was conducted using a transonic suck down intake rig (Fig. 2). The S-duct geometry used for this investigation was the high offset duct previously investigated by Zachos et al. [15] and GilPrieto et al. [16]. It is a circular section duct with a diffusion area of $A_{\text {out }} / A_{\text {in }}=1.52$, an offset to inlet diameter ratio of $H D_{\text {in }}=2.44$ and a length to inlet diameter ratio $L / D_{\text {in }}$ of 4.95 (Table 1 ). The geometry is based on the previous work of Garnier [14]. The PIV measurement plane (AIP) was $0.29 \mathrm{D}_{\text {in }}(36 \mathrm{~mm}$ ) downstream of the S-duct outlet (Fig. 2). The total pressure measurements were taken at a plane $0.5 \mathrm{D}_{\text {in }}(60.8 \mathrm{~mm})$ from the $\mathrm{S}$-duct outlet. Upstream of the $\mathrm{S}$ duct inlet, a constant diameter duct $\left(\mathrm{D}_{\text {in }}=121.6 \mathrm{~mm}\right)$ was used where boundary layer measurements were acquired at $0.9 \mathrm{D}_{\text {in }}(109.5 \mathrm{~mm})$ upstream the S-duct inlet. More detail of the experimental facility can be found in Zachos et al. [15].

The S-duct operating condition was defined in terms of the centerline inlet $\mathrm{M}_{\mathrm{in}}$ number. The inlet conditions were measured at the reference plane located $0.9 \mathrm{D}_{\text {in }}$ upstream of the S-duct inlet (Fig. 2). In this work the investigations were conducted at two inlet Mach numbers of 0.27 and 0.60 with a concomitant range in Reynolds number based on the inlet diameter from $\operatorname{Re}_{\mathrm{D}}=0.71 \times 10^{6}$ to $1.42 \times 10^{6}$ at nominal atmospheric pressure and temperature about $100 \mathrm{kPa}$ and $288 \mathrm{~K}$ respectively. The total pressure was measured using a Pitot probe with a $50 \mathrm{kPa}$ pressure transducer and a 16-bit DAQ card. Measurements were recorded for $10 \mathrm{~s}$ at a sample rate of $900 \mathrm{~Hz}$. The inlet reference Mach number was determined from the total pressure and wall static pressure measurements. For the typical operating condition of $\mathrm{M}_{\mathrm{in}}=0.27$ the measured boundary layer had a thickness $\left(\delta_{\mathrm{in}}\right)$ of $8 \mathrm{~mm}\left(\delta_{\mathrm{in}} / \mathrm{D}_{\mathrm{in}}=0.066\right)$. At $\mathrm{M}_{\mathrm{in}}=0.6, \delta$ was $7.5 \mathrm{~mm}$.

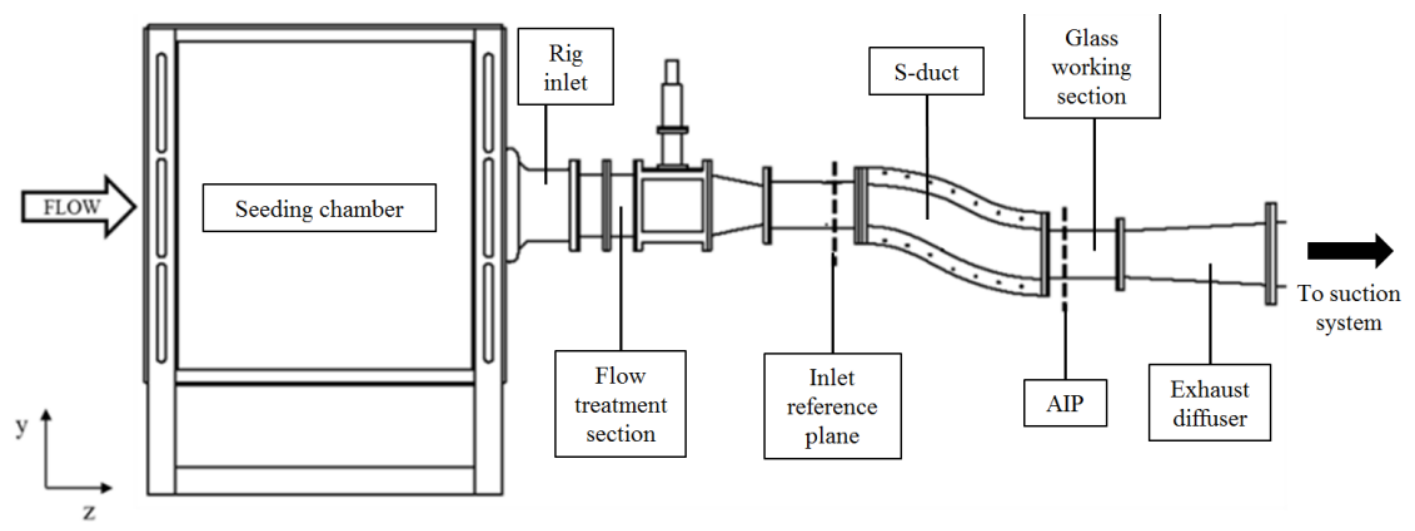

Fig. 2 General arrangement of the test rig [15] 


\section{Table 1 S-duct geometric parameters}

\begin{tabular}{cc}
\hline Parameter & High-offset Duct \\
\hline $\mathrm{D}_{\text {in }}(\mathrm{mm})$ & 121.6 \\
$\mathrm{D}_{\text {out }}(\mathrm{mm})$ & 150 \\
$\mathrm{~L}(\mathrm{~mm})$ & 601.6 \\
$\mathrm{~A}_{\text {out }} / \mathrm{A}_{\text {in }}$ & 1.52 \\
$\mathrm{~L} / \mathrm{D}_{\text {in }}$ & 4.95 \\
$\mathrm{H} / \mathrm{L}$ & 0.493 \\
$\mathrm{H} / \mathrm{D}_{\text {in }}$ & 2.44 \\
\hline
\end{tabular}

The uncertainty on the inlet Mach number was determined based on the uncertainties on the measured static and total pressures as well as ambient pressure and total temperature. The uncertainty assessment included both deterministic and stochastic elements. Including the effect of the tunnel calibration, the overall uncertainty on the operating Mach number is $0.27 \pm 0.010$ and $0.6 \pm 0.005$.

\section{B. Total pressure measurement}

The time averaged pressure measurements at the AIP are performed using a working section that can incorporate 6 pressure rakes and 6 static pressure taps. The section is able to rotate with a 5 degree increment. Ten total pressure probes with a tip diameter of $1.1 \mathrm{~mm}$ were mounted on a rake to measure the AIP total pressure field. Typically 1440 total pressure measurements points are taken at the AIP with a radial resolution of approximately $3.74 \mathrm{~mm}$ and circumferential resolution of $5^{\circ}$. Similarly 3 -hole probe rakes were used to measure the swirl angle across the AIP with 576 measurements points distributed as $8.75 \mathrm{~mm}$ in the radial and $5^{\circ}$ in the circumferential directions, respectively. The pressure measurements were acquired with 50kPa range low bandwidth pressure transducers. A sampling rate of $900 \mathrm{~Hz}$ and a sample number of 10000 were used to evaluate the time average total pressure and swirl distributions at the AIP.

\section{S-PIV methods}

An S-PIV system was used to measure the instantaneous 3 component velocity field at the AIP. The laser is a dual cavity pulsed Nd: YAG laser with a wave length of $532 \mathrm{~nm}$ and a maximum power of $200 \mathrm{~mJ}$ per pulse. An articulated laser arm was used to deliver a light sheet with an estimated thickness of $2 \mathrm{~mm}$ at the AIP. The laser arm was mounted onto a translation and rotation positioning system that allows the light sheet to be exactly placed and controlled within the region of interest. Di-ethyl-hexyl-sebacate (DEHS) was used as the seeding material. The 
seeding particles had an estimated diameter of $1 \mu \mathrm{m}$. Two TSI PowerView Plus 8MP rectangular sensors were used with AF 1.8/D Nikkor lenses with a focal length of 50mm at a stand-off distance of $600 \mathrm{~mm}$. The two CCD cameras were mounted symmetrically from each side of the rig at an angle of approximately 45 degrees. The aperture, focus and Scheimpflug arrangement were remotely controlled. The 3 component velocity field was calculated based on pairs of PIV images which were acquired with an interval of several micro-seconds. The overall repetition rate used to acquire the velocity field snapshots was $3.5 \mathrm{~Hz}$ and a total number of 1500 snapshots were captured for each case. This allowed the statistical assessment of the flow field and the distortion metrics. The magnification factor and the viewing direction of each camera are found by a calibration procedure using a spatial target plate marked by a rectangular grid of uniformly distributed circular dots spaced by $10 \mathrm{~mm}$. A 5-plane axial traverse method with an inter-plane spacing along the longitudinal axis of $0.375 \mathrm{~mm}$ was used to determine the spatial calibration factors.

The TSI Insight $4 \mathrm{G}^{\mathrm{TM}}$ was used for the calibration of the camera, the data acquisition and the post processing of the images. The potential error due to misalignment between the calibration target and the laser light sheet was corrected through a disparity correction also referred to as automaping procedure. The automaping process was done in an iterative way which includes multiple passes over 150 images for each data set. The cross correlation using a Nyquist grid was applied on the dewarped images from both cameras. A total of five iterations per data set was performed giving a mean mis-registration error around 3px [15]. Further details of the process are presented by Raffel et al. [34].

The pre-processing was used with a background subtraction method to remove the visual impact of the seeding accumulation, laser light reflection and static features in the pictures. A recursive Nyquist grid with 50\% overlaps was applied as a grid engine for the cross-correlation using a Fast Fourier Transform (FFT). A zeropadmask is used to increase the spot detection and a Gaussian engine was used to locate the correlation peak during the FFT. The first pass was executed over a 64px $\mathrm{x} 64 \mathrm{px}$ interrogation area with a $5 \mathrm{px} \times 5 \mathrm{px}$ validation pass. The second pass decreases the grid size to $32 \mathrm{px}$ x 32px with 50\% overlap window for the cross correlation. A second validation pass was performed including a vector smoothing process reducing to $10 \%$ the number of spurious vectors. After the validation, a total number of approximately 14,000 vectors for the AIP was obtained.

The overall uncertainty of the system was calculated following the analysis of Raffel et al. [34]. This analysis takes into account the particle image displacement, particle image diameter, the seeding density the quantization level and the background noise. The overall uncertainties for the in-plane and out of plane component of the velocity 
field were respectively $6 \%$ and $8 \%$. Zachos et al. [15] investigated the impact of the number of snapshots on the flow statistics at $\mathrm{M}_{\mathrm{in}}=0.27$. A data set of 2000 snapshots showed almost $0 \%$ change in average out of plane velocity and an increase of $0.60 \%$ in standard deviation the out of plane velocity relative to a set of 1000 snapshots. Relative to a data set of 1000 snapshot, a change in absolute average swirl angle and its standard deviation of $0.22 \%$ and $1.2 \%$ respectively were measured for a data set of 1500 snapshots. For this work, 1500 snapshots are considered sufficient to assess the dynamic flow field.

\section{Flow control characteristics}

The effect of flow control devices were experimentally investigated though different configuration of vortex generators (VG). Previous studies, based on conventional pressure distortion metrics, showed that main the variables which impact on the VG effectiveness are the VG height, axial position and circumferential extent $[21,29]$. In total, a set of nine configurations (Table 2) was selected to cover an exploration of the design space and provide an assessment of the effect of the design variables on the duct exit flow field at the AIP.

The vortex generators were made of $0.2 \mathrm{~mm}$ thick aluminum sheet with an orthogonal semi delta wing arrays. Two arrays of VGs were fixed on the surface of the S-duct in a co-rotating configuration at each side of the duct symmetry plane. A range of VG heights were assessed including $\mathrm{h} / \delta_{\text {in }}=0.63,0.88$ and 1.25 . The nominal inlet boundary layer was $\delta_{\mathrm{in}}=8 \mathrm{~mm}\left(\delta_{\mathrm{in}} / \mathrm{D}_{\mathrm{in}}=0.066\right)$. The aspect ratio $(\mathrm{c} / \mathrm{h})$ was 0.25 with a constant toe angle $\left(\beta_{v g}\right)$ of $16^{\circ}$. Three circumferential extents $\left(\theta_{s}\right)$ (Fig. 3) of $67.5^{\circ}, 97.5^{\circ}$ and $157.5^{\circ}$ were investigated in which the number of vortex generators was changed. These circumferential extents correspond to 10,14 and 22 VGs for a constant angular lateral spacing $\alpha_{s}$ of $15^{\circ}$. Two additional cases were investigated with an $\alpha_{s}$ of $10^{\circ}$ and $20^{\circ}$ over a circumferential extant of $105^{\circ}$ and $90^{\circ}$, respectively. The VG axial position is defined by the distance from the datum separation location which was determined from oil flow visualization (Fig. 4). Although this is mainly a qualitative method to evaluate separation, it is similar to previous work in this area [3,11]. For the datum configuration, the nominal centerline separation arises at an axial distance of $187 \mathrm{~mm}$ from the inlet. The first VG array was placed at the inlet of the duct at a distance of $\Delta \mathrm{x} / \delta_{\text {in }}=20.8(166 \mathrm{~mm})$ upstream of the separation. The second and third axial positions were chosen at an upstream distance of $\Delta \mathrm{x} / \delta_{\text {in }}=14.0(112 \mathrm{~mm})$ and $4.63(37 \mathrm{~mm})$, respectively. 

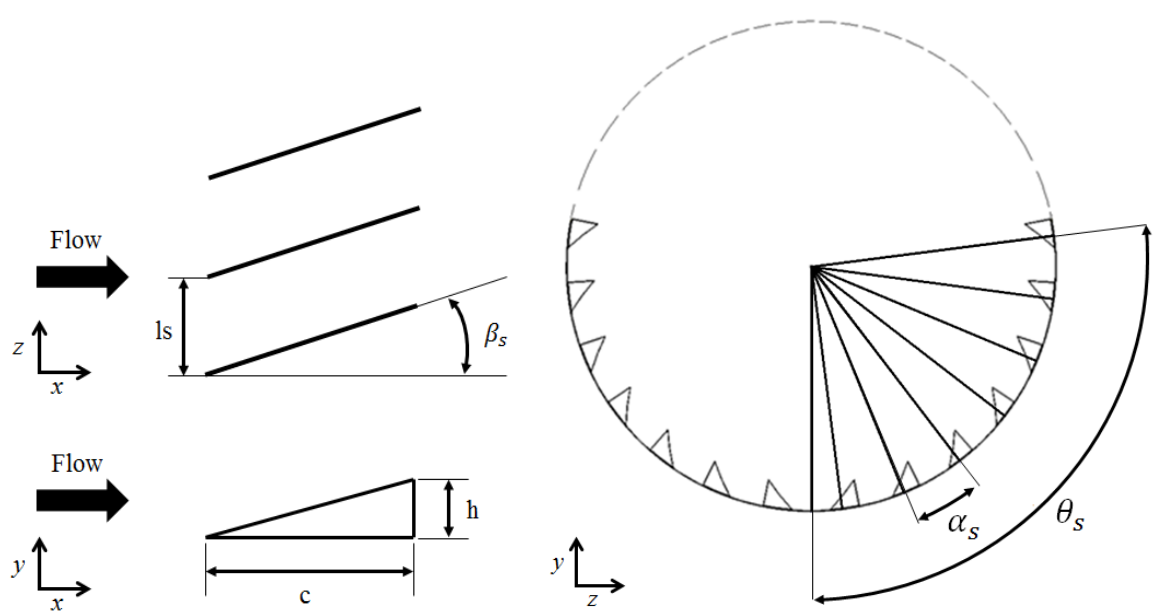

Fig. 3 Vortex generator schematic and nomenclature

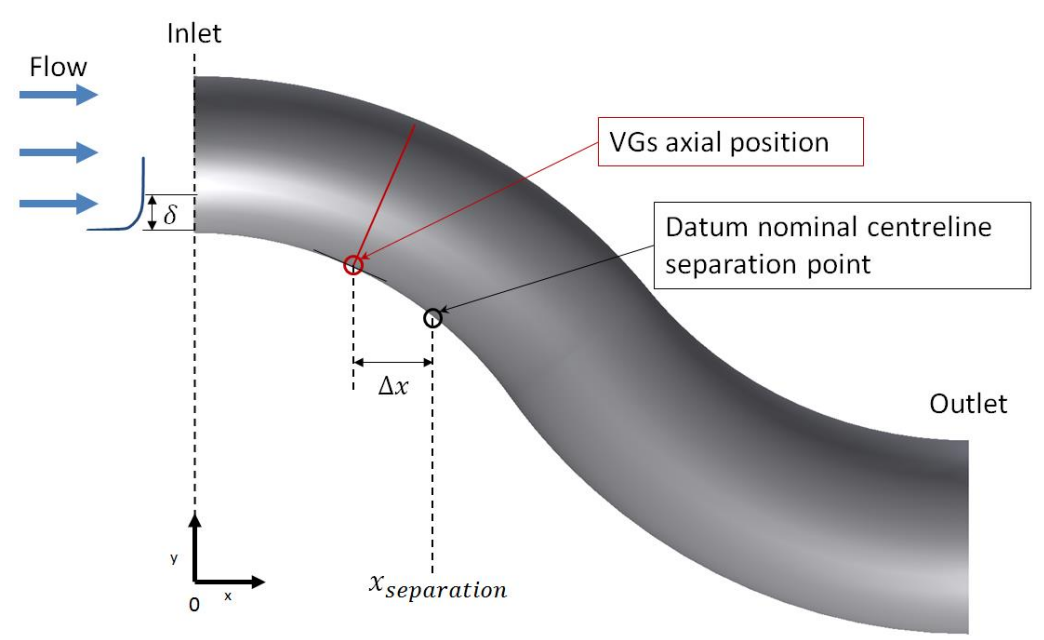

Fig. 4 VG axial position definitions

\section{E. Distortion assessment for S duct}

Several distortion descriptors are used to quantify and evaluate the effect of vortex generators on the flow field at the AIP. The duct efficiency is evaluated with the pressure recovery (PR) which is the ratio between the area weighted average total pressures at the AIP $\left(\left\langle{\overline{P_{0}}}_{A I P}\right\rangle\right)$ and the reference pressure $\left(P_{0, r e f}\right)$ measured at the plane located $0.9 \mathrm{D}_{\text {in }}$ upstream of the S-duct inlet (Fig. 2).

$$
P R=\frac{\left\langle{\overline{P_{0}}}_{A I P}\right\rangle}{P_{0, r e f}}
$$


Table 2 Summary of VG configurations

\begin{tabular}{ccccccc}
\hline Case & $\theta_{s}\left(^{\circ}\right)$ & $\begin{array}{c}\text { number of } \\
\text { VG }\end{array}$ & $\begin{array}{c}\text { axial } \\
\text { position } \\
\left(\Delta \mathrm{x} / \delta_{\text {in }}\right)\end{array}$ & $\begin{array}{c}\text { VG } \\
\text { height } \\
\left(\mathrm{h} / \delta_{\text {in }}\right)\end{array}$ & $\begin{array}{c}\text { VG } \\
\text { spacing } \\
\alpha_{s}\left(^{\circ}\right)\end{array}$ & $\begin{array}{c}\beta_{s} \\
\left({ }^{\circ}\right)\end{array}$ \\
\hline VG1 & 67.5 & 10 & 4.63 & 0.88 & 15 & 16 \\
VG2 & 97.5 & 14 & 4.63 & 0.88 & 15 & 16 \\
VG3 & 157.5 & 22 & 4.63 & 0.88 & 15 & 16 \\
VG4 & 97.5 & 14 & 4.63 & 0.63 & 15 & 16 \\
VG5 & 97.5 & 14 & 14 & 0.63 & 15 & 16 \\
VG6 & 67.5 & 10 & 20.8 & 0.63 & 15 & 16 \\
VG7 & 105 & 22 & 14 & 0.63 & 10 & 16 \\
VG8 & 90 & 10 & 14 & 1.25 & 20 & 16 \\
VG9 & 97.5 & 14 & 4.63 & 1.25 & 15 & 16 \\
\hline
\end{tabular}

The relative loss in total pressure ratio is also characterized by:

$$
\Delta \eta=\left(\frac{(1-P R)_{V G}-(1-P R)_{\text {datum }}}{(1-P R)_{\text {datum }}}\right) 100
$$

The DC (60) is also conventionally considered to evaluate the level of total pressure distortion at the AIP. It is defined as the difference between the average total pressure $\left\langle{\overline{P_{0}}}_{A I P}\right\rangle$ and the lowest mean total pressure on a $60^{\circ}$ sector $\left\langle{\overline{P_{060^{\circ}}}}\right\rangle$ non-dimentionalized by the mean dynamic head $q$ of the AIP [35]. The dynamic head is calculated from the time-averaged wall static pressure measured with a circumferential resolution of $5^{\circ}$ at the AIP.

$$
\operatorname{DC}(60)=\frac{\left\langle{\overline{P_{0}}}_{A I P}\right\rangle-\left\langle{\overline{P_{0}}}_{60^{\circ}}\right\rangle}{q}
$$

Several descriptors were proposed by the Society of Automotive Engineers (SAE) in order to characterize the distorted flow-field for swirl non-uniformities [4]. The AIP is discretized into several rings and each descriptor is evaluated at each radial position based on the swirl angle distribution. A two-per-revolution swirl pattern is illustrated in Fig. 5 as an example for the $\mathrm{i}^{\text {th }}$-ring at the AIP. Positive and negative sector swirls, $S S_{i, k}^{+}$and $S S_{i, k}^{-}$, are 
defined as the mean swirl-angle value in each of the swirling regions. The circumferential extent, $\theta_{i, k}^{+}$and $\theta_{i, k}^{-}$, define the size of these swirling regions. The swirl angle is considered positive in the counter-clock wise direction when the AIP is view from downstream.

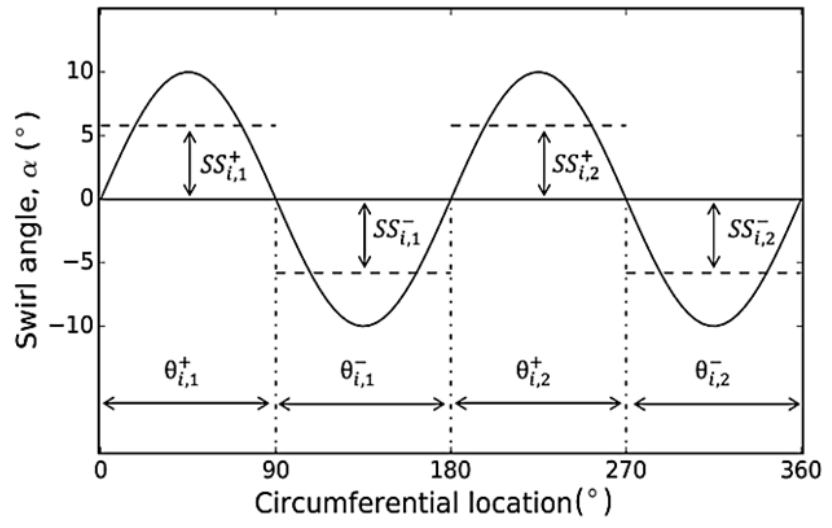

Fig. 5 Twice per revolution swirl distortion pattern from Gil-Prieto[16] based on SAE[4]

The swirl intensity (SI) calculates the average absolute swirl angle for a given ring (Eq.4). The swirl directivity (SD) represents the overall sense of rotation of the swirling flow in the ring (Eq. 5). The swirl pairs (SP) indicates the number of swirl pairs relative to the region in the ring which encloses the highest absolute swirl angle content: $\max \left(S S_{i, k}^{+} \theta_{i, k}^{+},\left|S S_{i, k}^{-} \theta_{i, k}^{-}\right|\right)[4]$

$$
\begin{gathered}
S I(i)=\frac{\sum_{k=1}^{m} S S_{i, k}^{+} \cdot \theta_{i, k}^{+}+\sum_{k=1}^{m}\left|S S_{i, k}^{-}\right| \cdot \theta_{i, k}^{-}}{360} \\
S D(i)=\frac{\sum_{k=1}^{m} S S_{i, k}^{+} \cdot \theta_{i, k}^{+}+\sum_{k=1}^{m} S S_{i, k}^{-} \cdot \theta_{i, k}^{-}}{\sum_{k=1}^{m} S S_{i, k}^{+} \cdot \theta_{i, k}^{+}+\sum_{k=1}^{m}\left|S S_{i, k}^{-}\right| \cdot \theta_{i, k}^{-}} \\
S P(i)=\frac{\sum_{k=1}^{m} S S_{i, k}^{+} \cdot \theta_{i, k}^{+}+\sum_{k=1}^{m}\left|S S_{i, k}^{-}\right| \cdot \theta_{i, k}^{-}}{2 \cdot \operatorname{Max}\left\{S S_{i, k}^{+} \cdot \theta_{i, k}^{+},\left|S S_{i, k}^{-} \cdot \theta_{i, k}^{-}\right|\right\}_{k=1, \ldots, m}}
\end{gathered}
$$

The swirl descriptors SD and SP are used to describe the shape of the swirl pattern in the ring evaluated (Fig. 6, Fig. 7). The AIP is divided in 9 equi-spaced rings with a circumferential resolution of $5^{\circ}$ equivalent to 72 rakes. The descriptors SI(i), SP(i) and SD(i) calculated for each rings are area weighted averaged to obtain one value of SI, SP 
and SD per snapshot. The descriptors are then averaged in time to obtain the mean descriptors $\mathrm{SI}_{\text {mean }}, \mathrm{SP}_{\text {mean }}$ and $\mathrm{SD}_{\text {mean }}$

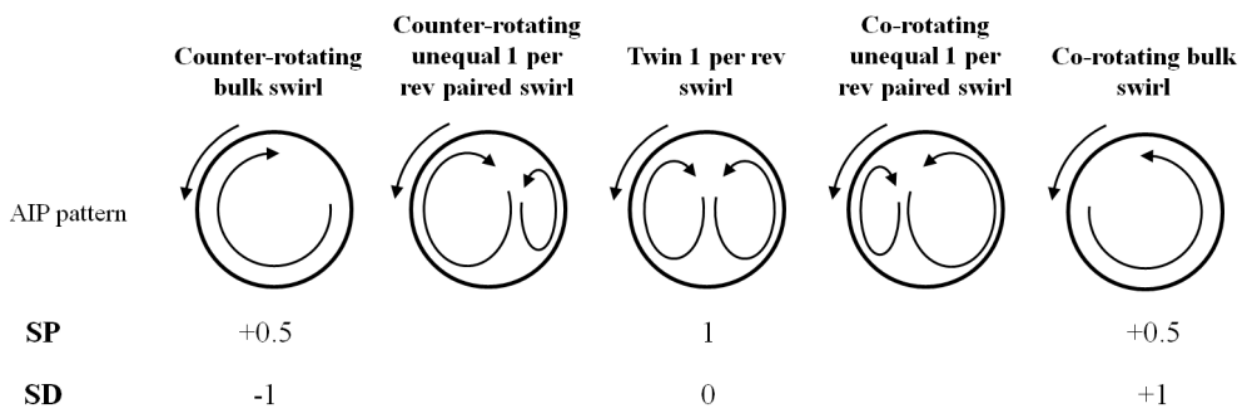

Fig. 6 Swirl Pairs and Swirl Directivity range for one-per-revolution swirl patterns based on SAE [4]

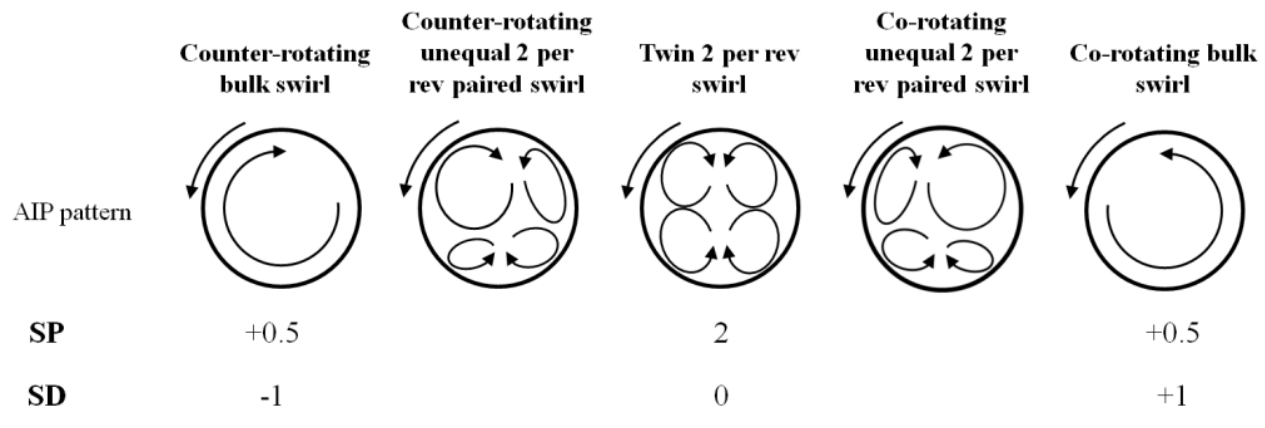

Fig. 7 Swirl Pairs and Swirl Directivity range for multiple-per-revolution swirl patterns based on SAE [4]

\section{Results}

The experimental work combines both S-PIV measurements and pressure measurements at the exit of the S-duct. The key pressure distortion descriptors have shown a dependency on the Mach number. Therefore the impact of seven vortex generators (VG) configurations on the steady total pressure field is assessed for an inlet Mach number of 0.27 and 0.60 . The S-PIV measurements are used to assess the impact of the nine VG configurations on the unsteady velocity and swirl flow field at the AIP for an inlet Mach number of 0.27.

\section{A. Effect of vortex generators on the mean flow field}

\section{Total pressure and swirl results}

The mean flow field at the AIP for the datum configuration presents non-uniformities in both total pressure and pressure based swirl angle distributions (Fig. 8). The total pressure field demonstrates losses on the lower central part of the AIP caused by the interaction between the classical secondary flows and centerline flow separation at the first bend of the S-duct. The adverse pressure gradient established at the second bend of the S-Duct causes a second 
loss region at the top of the AIP (Fig. 8a). However, surface oil flow visualizations did not provide evidence of a separation in this region. The mean swirl angle distribution indicates the presence of the well-known symmetric vortex pair at the AIP, which was observed by Wellborn et al. [3]. The variation from Min=0.27 to Min=0.60 does not influence the shape of the mean total pressure and swirl angle fields at the AIP. However the pressure deficit in the loss region increases with the inlet Mach number with a reduction of $\operatorname{PR}\left(\left\langle{\overline{P_{0}}}_{A I P}\right\rangle / P_{0, \text { ref }}\right)$ from 0.990 to 0.958.

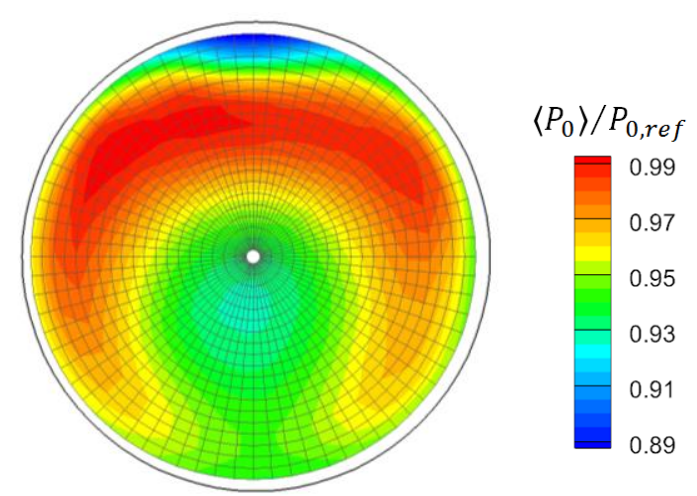

a)

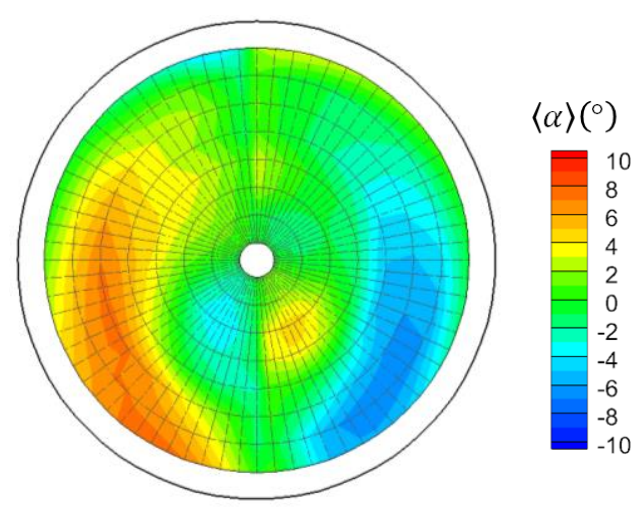

b)

Fig. 8 Total pressure recovery (a) and swirl angle (b) field at the AIP for Min $=0.6$

Vortex generators (VGs) are designed to reduce the unsteady distortion of the flow at the outlet of the S-duct by the control of the secondary flows and the suppression of separated flow regions inside the S-duct. The VG configurations have a substantial effect on the distributions of total pressure ratio $\left(\left\langle P_{0}\right\rangle / P_{0, \text { ref }}\right)$ at the AIP (Fig. 9). The total pressure loss region in the lower center part for the datum configuration is replaced by a higher uniform total pressure flow field. However concentrated loss regions which differ in shape, intensity and extent from one case to another are generated. An initial assessment of the impact of the VGs is considered in terms of average total pressure ratio (PR) (Fig. 10).

For both inlet Mach numbers (0.27 and 0.60) there is an overall increase in PR for all the VG configurations investigated (Fig. 10a). The effectiveness of the VGs is proportionally the same for both Mach numbers with a similar reduction in loss relative to the datum case $(\Delta \eta)$ (Fig. 10b). The best performance is provided by VG5 where there is a maximum increase in PR. At Min $=0.27$, the PR increases from 0.99 to 0.993 while at Min=0.60, the PR increases from 0.958 to 0.970 . VG5 is a configuration with a low profile VG array which is positioned half of the distance between the inlet and the separation point $\left(\theta_{s}=157.5^{\circ}, h / \delta=0.63, \Delta x / \delta_{\text {in }}=14\right)$. Conversely, although all the configurations provided an improvement in PR, $\operatorname{VG} 9\left(\theta_{s}=97.5^{\circ}, h / \delta=1.25, \Delta x / \delta_{\text {in }}=4.63\right)$ produced the smallest relative benefit with an improvement in PR from 0.958 to 0.965 at $\mathrm{M}_{\mathrm{in}}=0.6$. In general, with the exception 
of VG4 $\left(\theta_{s}=97.5^{\circ}, h / \delta_{\text {in }}=0.63, \Delta x / \delta_{\text {in }}=4.63\right)$, the configurations with the lower height VGs provided the best improvements in PR (Fig. 10).

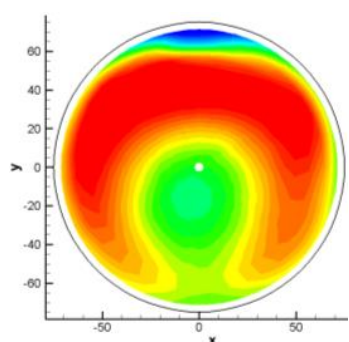

a) Datum

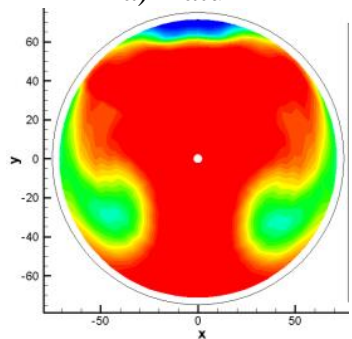

e) VG 5

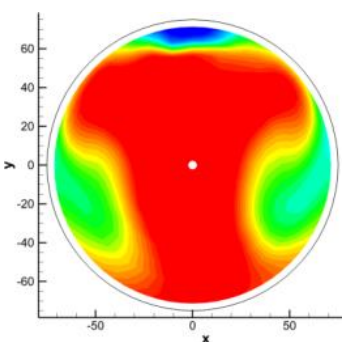

b) VG 1

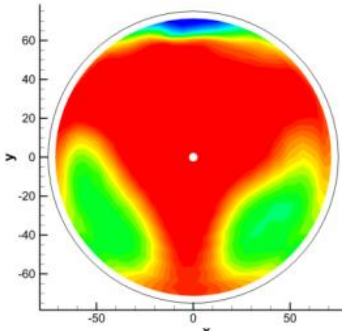

f) $\operatorname{VG}^{x} 6$

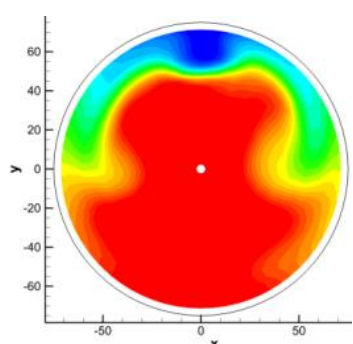

c) $\mathrm{VG}^{2}$

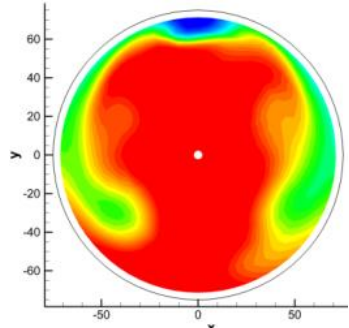

g) VG 7

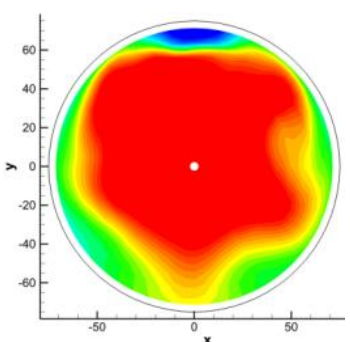

d) $\mathrm{VG}^{4}$

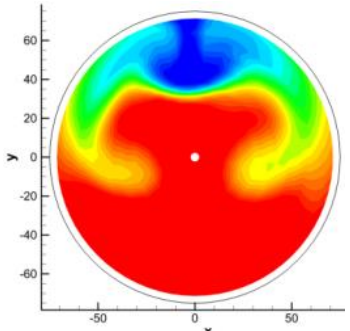

h) $V^{x} 9$

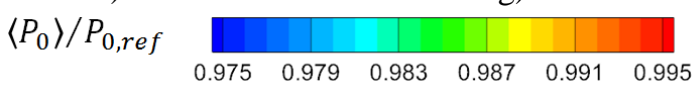

Fig. 9 Time averaged total pressure distributions at $M_{\text {in }}=0.27$

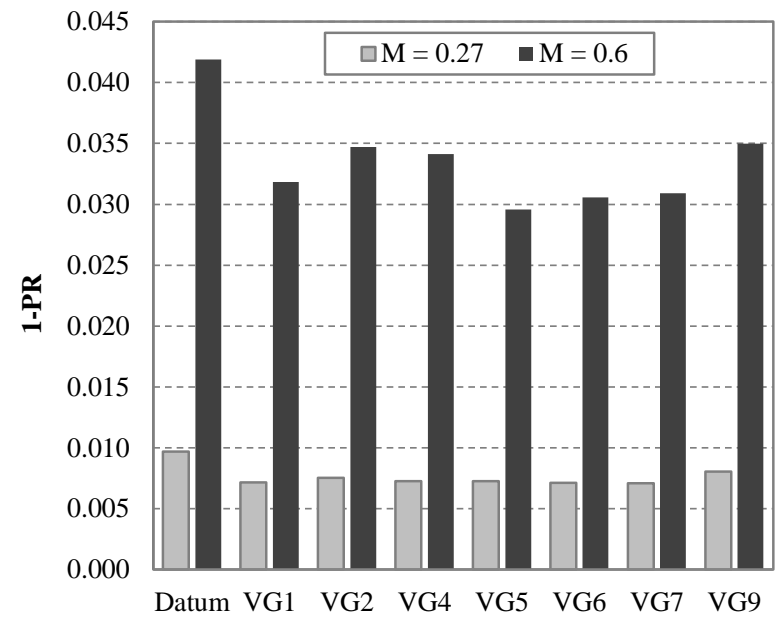

a)

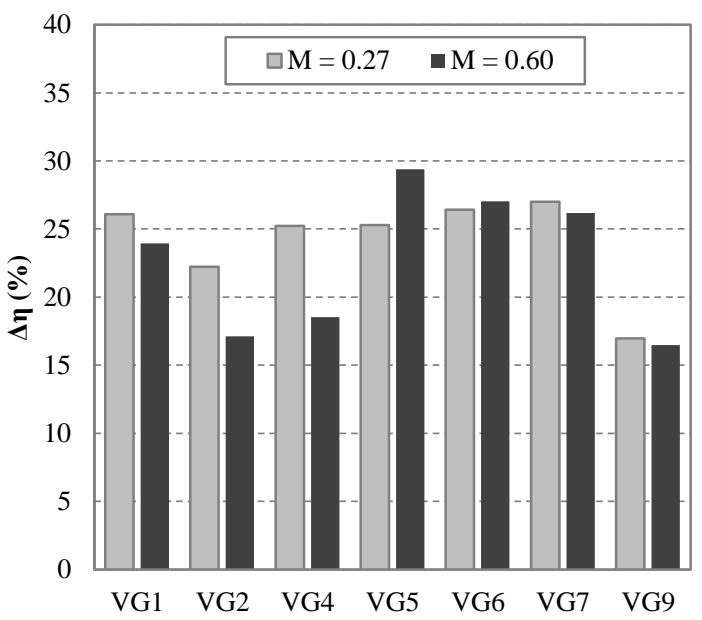

b)

Fig. 10 Time averaged total pressure recovery (a) and relative change in total pressure loss compare with the datum configuration (b) at the AIP 


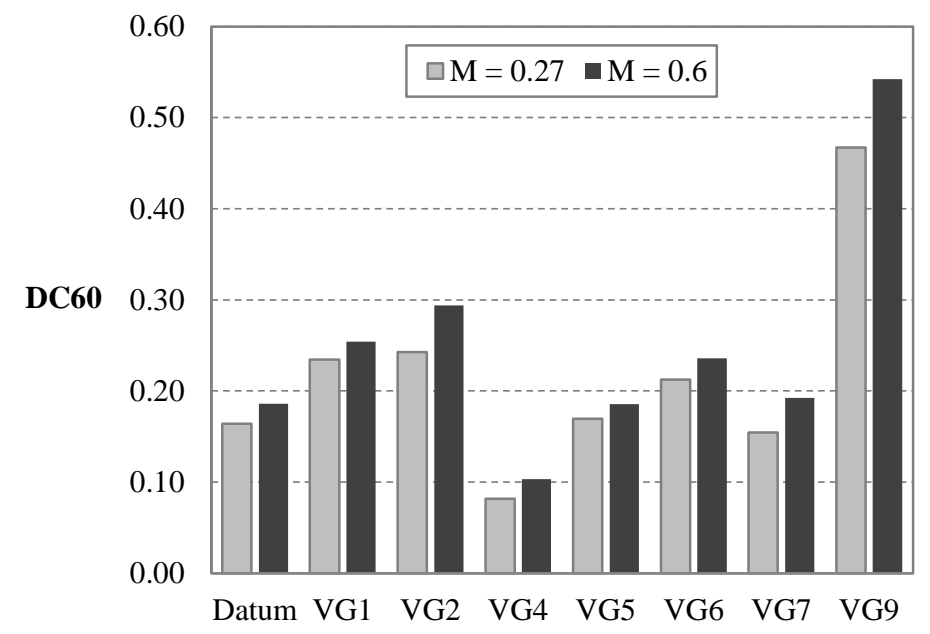

Fig. 11 Effect of VGs the DC60 steady state distortion

The Mach number effect is similar for both the datum configuration and the VG configurations with a decrease in PR when the inlet Mach number increases from 0.27 to 0.60 . For the datum configuration, a reduction in PR from 0.990 to 0.958 , which corresponds to a decrease of 3.3\%, arises when the inlet Mach number increases from 0.27 to 0.6. The reduction in PR for the VG flow control cases is between $2.4 \%$ and $2.7 \%$ when the Mach number increases from 0.27 to 0.60 which highlights that PR for the VG configurations are slightly less sensitive to $M_{\text {in }}$ relative to the datum case.

The DC60 descriptor is a means to quantify the impact of the VG configurations on the mean total pressure distortion levels (Fig. 11). Unlike the total pressure recovery, the addition of the VGs can both increase and reduce the DC60 relative to the datum case. However, it is also noteworthy, that the definition of the DC60 parameter means that it is sensitive to the location of the main loss region. For example, a pressure deficit region at the center of the AIP would provide a smaller DC60 compared to the case in which the same low-pressure flow is radially positioned in a sector of the AIP.

The configurations VG1 and VG2 are located near the nominal axial separation point and have the same VG height $\left(h / \delta_{\text {in }}=0.88, \Delta x / \delta_{\text {in }}=4.63\right)$. The only difference between these VG configurations is the number of equi-spaced VGs, with a concomitant increase in the circumferential extent covered by the VGs from $\theta_{s}=67.5^{\circ}$ to $97.5^{\circ}$. For VG1, two loss regions appear at each side of the AIP which are associated with the presence of a pair of contra rotating vortices (Fig. 9b). This pattern was also observed in the flow control investigation by Delot et al. [32] and Reichert et al. [11]. VG2 total pressure flow field (Fig. 9c) is similar to that reported by Anderson et al. [22] and 
Jirasek [29] where the total pressure losses are located near the wall at the top sector of the AIP. For VG1 and VG2, the increase of the extent covered by the VGs $\left(\theta_{s}\right)$ from $67.5^{\circ}$ to $97.5^{\circ}$ moves the loss region toward the upper periphery of the AIP. However, a high value of $\theta_{s}$ can increase the adverse pressure gradient due to the second bend of the duct and promote flow separation at the top of the AIP (Fig. 9c). This is demonstrated in VG2, which shows deterioration in both DC60 (Fig. 11) and PR (Fig. 10) due to the substantial loss at the upper sector of the section. VG4 presents the best characteristics in terms of DC60 (Fig. 11) with a value of 0.10 at high inlet Mach number which represents a decrease of 50\% compared with the datum configuration. This is explained by the reduction in extent of the loss regions that are circumferentially spread on the periphery of the AIP (Fig. 9d). VG5 and VG7 are both located half the distance between the separation point and the inlet $\left(\Delta x / \delta_{\text {in }}=14\right)$. A similar circumferential extent covered by the VGs is used for both configurations, with $\theta_{s}=97.5$ and $\theta_{s}=105$ for VG5 and VG7 respectively. The only change in design variable is their lateral spacing and thus the number of VGs is 14 and 22. VG5 and VG7 pressure recovery are respectively 0.970 and 0.969 for $\mathrm{M}_{\mathrm{in}}=0.6$ (Fig. 10). Therefore the increase of the number of VGs does not significantly affect PR for this case. VG6 comprises 10 low profile VGs $\left(\theta_{s}=\right.$ $\left.67.5^{\circ}, h / \delta_{i n}=0.63\right)$ located at the inlet of the S-duct $\left(\Delta x / \delta_{i n}=20.8\right)$. In this case two distinct pressure losses are generated at each side of the AIP (Fig. 9f) increasing the DC60 up to 0.24 for $\mathrm{M}_{\mathrm{in}}=0.6$. VG9 $\left(\theta_{s}=97.5, h / \delta_{\text {in }}=\right.$ $1.25, \Delta x / \delta_{\text {in }}=4.63$ ) presents the highest level of distortion with a DC60 about three times greater compared with the datum configuration for $\mathrm{M}_{\mathrm{in}}=0.6$ (Fig. 11). This is caused by the strong secondary losses generated at the top AIP by the tall VGs $\left(h / \delta_{\text {in }}=1.25\right)$.

Therefore the introduction of VGs can significantly modify the total pressure field at the AIP. Overall, a positive impact of up to $1.28 \%$ is observed for the pressure recovery ratio which can directly improve the overall propulsion efficiency. DC60 tends to be very sensitive to the configuration used. DC60 can be reduced by half with VG4 that comprise low profile VGs placed near the nominal centerline separation $\left(\theta_{s}=97.5^{\circ}, h / \delta_{\text {in }}=0.63, \Delta x / \delta=4.63\right)$. S-PIV results

The datum and the nine vortex generator configurations were investigated using the S-PIV measurement system to obtain the 3 component velocity field at the AIP. The time averaged and some unsteady aspects of the flow field for the datum configuration were previously investigated by Zachos et al. [15] for the same S-duct configuration $(\mathrm{H} / \mathrm{D}=2.44)$ at an inlet Mach number range between 0.27 and 0.60 . The impact of the Mach number on the streamwise velocity field $\langle w\rangle /\left\langle\bar{w}_{A I P}\right\rangle$ was found to be minor. Zachos et al. [15] also found that the Mach number 
had a very limited impact on both the time averaged and fluctuating swirl angle flow field. Therefore, only a single inlet Mach number of 0.27 is considered in the present S-PIV investigation for the swirl distortion assessment.

For the datum configuration, the combination of the separation region that occurs at the first bend of the S-duct and the secondary flow field promoted by the curvature generates a deficit in streamwise velocity at the AIP (Fig. 12). This region of low streamwise velocity is associated with a loss in total pressure (Fig. 9a). The analysis of the time averaged swirl angle $(\alpha)$ distribution for the datum configuration (Fig. 13a) shows a pair of counter-rotating swirling regions on both side of the symmetry axis of the AIP with absolute values of $\alpha$ as high as $15^{\circ}$ are measured near the lower sector of the AIP. However, the low streamwise velocity region at the center of the AIP is associated with relatively low absolute swirl angles. The time averaged in-plane velocity streamlines for the datum configuration (Fig. 12a) indicates the well-known symmetrical pair of counter rotating vortices observed by Wellborn et al [3].

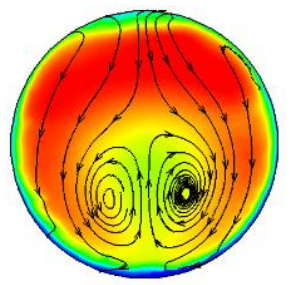

a) Datum

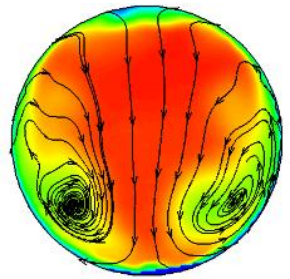

f) VG5

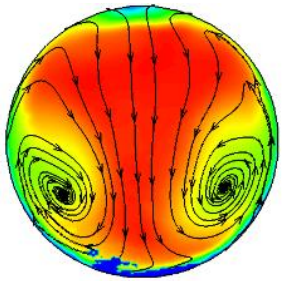

b) VG1

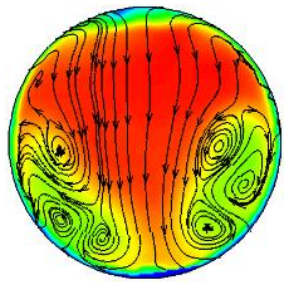

g) VG6

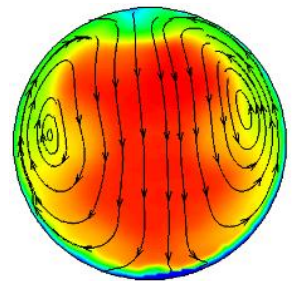

c) VG2

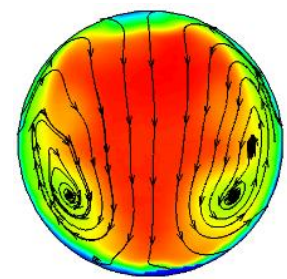

h) VG7

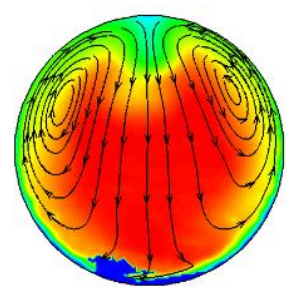

d) VG3

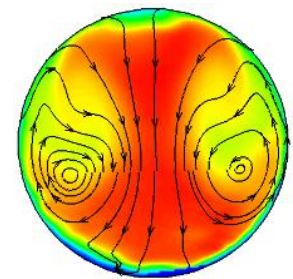

i) VG8

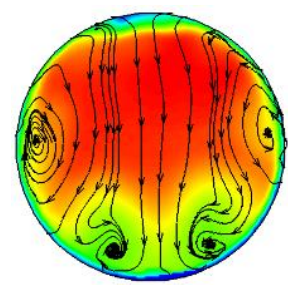

e) VG4

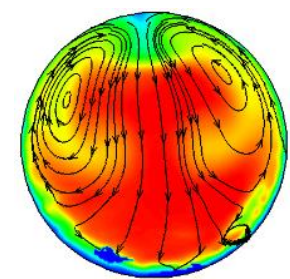

j) VG9

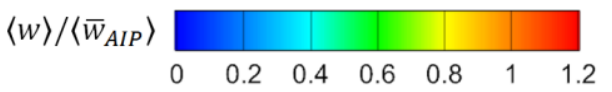

Fig. 12 Non-dimensional time averaged out of plane velocity with in-plane stream lines from S-PIV at $\mathbf{M}_{\mathrm{in}}=\mathbf{0 . 2 7}$

Relative to the datum configuration, all the VG configurations have a notable impact on the out of plane velocity flow field at the AIP (Fig. 12). The introduction of vortex generators alters the shape, extent and intensity of both the velocity deficit and the swirling structures. The regions of low-streamwise velocity are co-located with the low total pressure regions (Fig. 9). The vortical structures inferred from total pressure measurement are confirmed by the swirl angle distribution (Fig. 13) and the in-plane velocity streamline (Fig. 12) for the vortex generator configurations. For all the VGs configurations, the flow topology is modified with a suppression of the central loss 
region visible in the datum case. Instead a high velocity core is associated with a bulk downward pitching of the mainly attached flow field at the center part of the AIP (Fig. 14). As a result the naturally occurring secondary vortices are modified into two separate swirling regions which have migrated toward the periphery of the AIP.

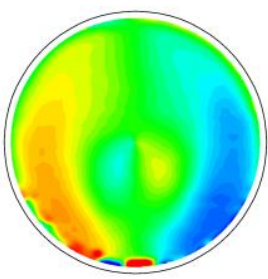

a) Datum

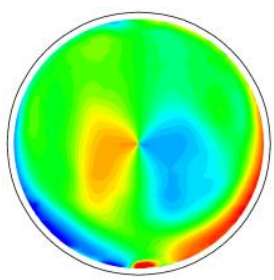

f) VG5

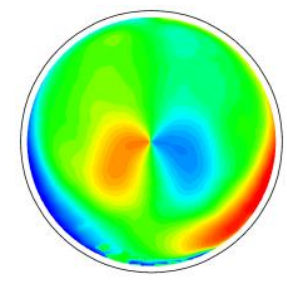

b) VG1

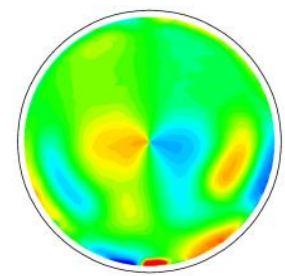

g) VG6

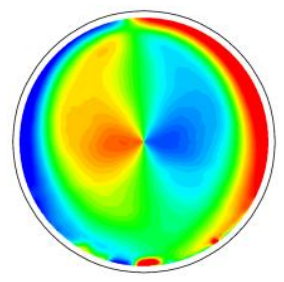

c) VG2

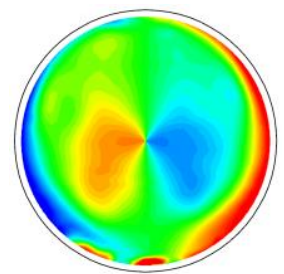

h) VG7

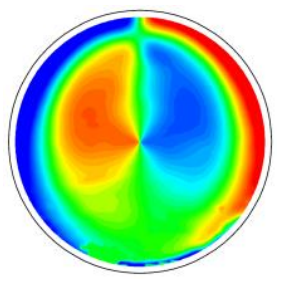

d) VG3

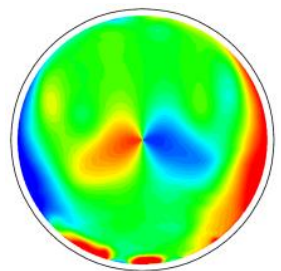

i) VG8

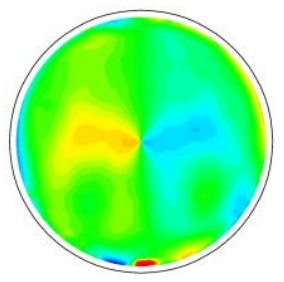

e) VG4

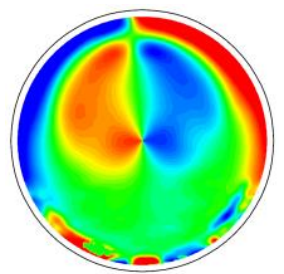

j) VG9

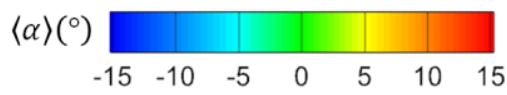

Fig. 13 Time averaged swirl angle from S-PIV at $M_{\text {in }}=0.27$

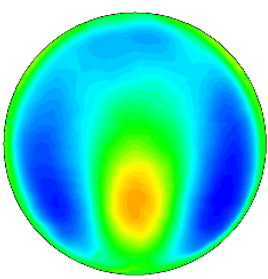

a) Datum

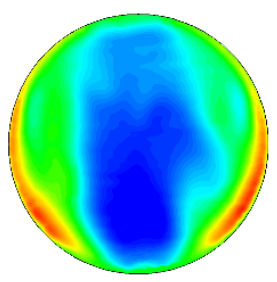

f) VG5

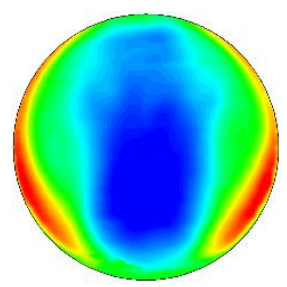

b) VG1

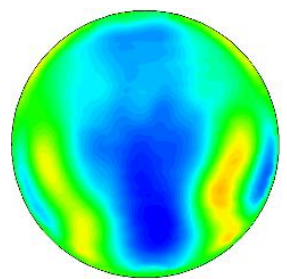

g) VG6

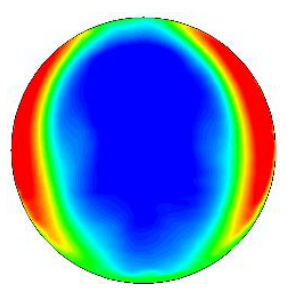

c) VG2

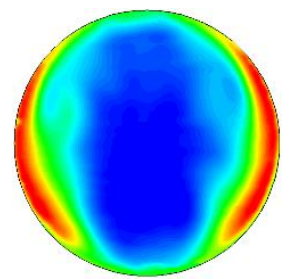

h) VG7

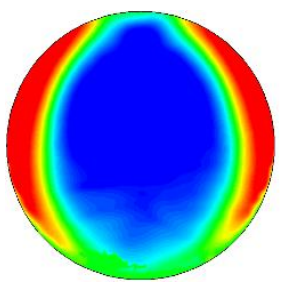

d) VG3

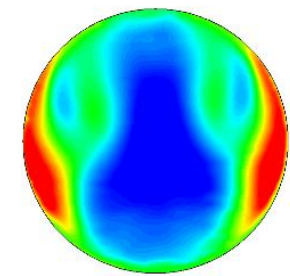

i) VG8

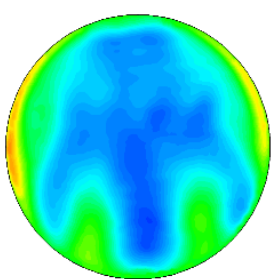

e) VG4

$\langle v\rangle /\left\langle\bar{w}_{A I P}\right\rangle$

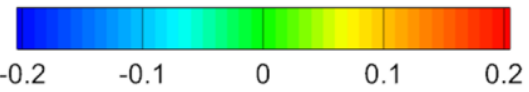


Fig. 14 Non-dimensional time averaged vertical velocity from S-PIV at $M_{i n}=0.27$

The notable effect of the VGs is evident in the time averaged swirl distribution (Fig. 13). Apart from cases 4 and 6, the swirl distribution pattern is made of two pairs of positive and negative swirl angle regions. High levels of swirl angle are present at the center of the AIP as well as near the walls. This is due to the presence of a pair of vortices which rotate in the opposite direction compared to the datum flow. The position and strength of these vortex pairs are sensitive to the design conditions of the VGs. The time-averaged flow fields for VG4 and VG6 present lower levels of swirl angle than for the other VG configurations at the AIP. However, multiple regions of opposite swirl angle which characterize more than one pair of vortices can be noticed (Fig. 13e and g). For example, the inplane velocity streamlines for VG6 (Fig. 12g) show three contra-rotating vortices associated with the low velocity regions located at both side of the symmetry plane. As a result, the swirl angle distribution is disrupted into weaker, smaller regions compare with the other VG configurations (Fig. 13g). The rotation sense of the central vortex pair suggests that the naturally occurring secondary flows are not totally suppressed by the vortex generators for VG4 and VG6 (Fig. 12e and g).

Therefore, as for the total pressure flow field, the vortex generators significantly modify the streamwise velocity. The in-plane velocity field is also substantially affected, which ultimately modifies the swirl angle distribution at the AIP. In all cases the datum secondary flows are suppressed. As a result, new pairs of vortices are generated at the AIP that relocate the loss regions toward the periphery of the AIP (Fig. 12). These changes typically result in a pitch down region (Fig. 14) which suggests the suppression of the centerline streamwise separation region observed for the datum configuration. However substantially higher levels of mean swirl angle (Fig. 13) are generated by the new secondary flows.

\section{B. Vortex generator effect on the unsteady flow field}

One of the keys benefits of S-PIV is the ability to obtain synchronous data across the full AIP. This allows the distorted flow field to be evaluated at each snapshot and thereby enables a statistical assessment of the instantaneous dynamic flow distortion. Although the time averaged solution of the flow field at the AIP is symmetric, previous experimental studies have demonstrated the unsteady nature of the flow field [15]. The time averaged distribution of the swirl angle (Fig. 13) provides a misleading message about the importance of the high swirl angle regions at the AIP. For the datum configuration, the region of relatively low mean out of plane velocity (Fig. 12a) located near the bottom center sector of the AIP is associated with moderate time averaged swirl angle values from $-6^{\circ}$ to $6^{\circ}$ (Fig. 
13a). However, high swirl unsteadiness are present on the lower sector of the AIP with values of the standard deviation of swirl $\left(\sigma_{\alpha}\right)$, as high as $15.8^{\circ}$ (Fig. 15a). Therefore the inner section of the AIP is also subject to large swirl values due to high $\sigma_{\alpha}$. Gil-Prieto et al. [16] also reported the presence of peak values in absolute swirl angle higher than $25^{\circ}$ along a circumferential locus of $\mathrm{r} / \mathrm{R}=0.32$.

The use of VGs significantly reduces the dynamic levels of the swirl angle for all the controlled-flow cases (Fig. 15). However some configurations demonstrate more fluctuation in $\alpha$ and present different flow topologies relative to the other VG cases. For example, the region of high unsteady $\sigma_{\alpha}$ for VG1, VG5 and VG7 (Fig. 15a, f and h) are associated with regions of low out of plane velocity on each side of the AIP (Fig. 12a, f and h). These regions of fluctuating swirl are separated by a relatively steady flow field located on the symmetry axis of the AIP. For example for VG1 a region with $\sigma_{\alpha}=1^{\circ}$ and $\alpha=0^{\circ}$ is present along the symmetry axis (Fig. 15b). Therefore, the two vortical regions on each side of the AIP generated by the new secondary flow field for VG1, VG5 and VG7 are confined in their respective loss regions. As a result, there is a limited interaction between the two loss regions on each side of the AIP which contribute to the global reduction in unsteadiness of the flow field compared with the datum configuration. VG 4 and VG6 do not seem to generate enough secondary flows to totally suppress the separation region due to the curvature of the duct. Both configurations presented multiple pairs of vortices for the time averaged solution with relatively low pitching down velocity levels in the central region compared with the other VG configurations (Fig. 14). As a result, the swirl unsteadiness is increased on the bottom sector of the AIP with values of $\sigma_{\alpha}$ of $8^{\circ}$ and $11^{\circ}$ for VG4 and VG6 respectively.

VG2 and VG3 present similar patterns with the unsteady swirl region circumferentially distributed near the periphery of the AIP (Fig. 15c, d). For these configurations, the VGs generate strong secondary flows resulting in high swirl angle value near the top periphery of the AIP (Fig. 13c, d). However the core flow of the AIP is stabilized with $\sigma_{\alpha}$ values between $0^{\circ}$ and $3^{\circ}$ (Fig. $15 \mathrm{c}, \mathrm{d}$ ). The convergence of the large swirling structure at the top of the AIP also generates peak levels of instabilities reaching almost $22^{\circ}$ associated with a loss in both mean stream-wise velocity (Fig. 12c, d) and mean total pressure (Fig. 9c). VG8 shows relatively low levels of $\sigma_{\alpha}$ with fluctuations in swirl angle present in the upper sector of the AIP (Fig. 15i). Overall the average peak swirl unsteadiness for all the VG cases is $61 \%$ lower than the datum configuration. 


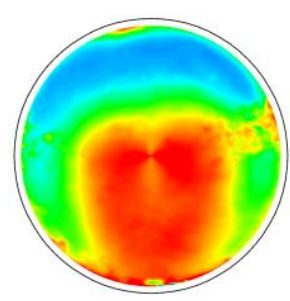

a) Datum

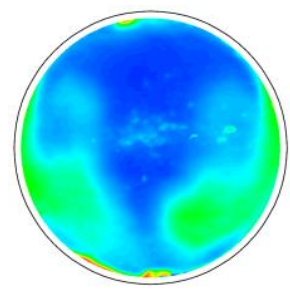

f) VG5

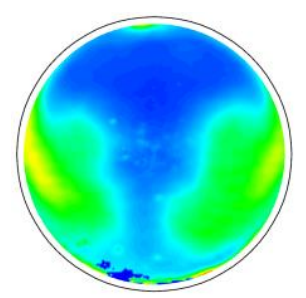

b) VG1

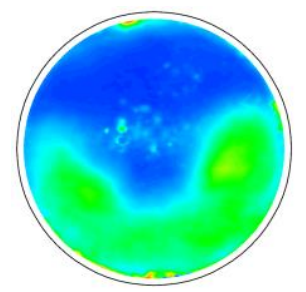

g) VG6

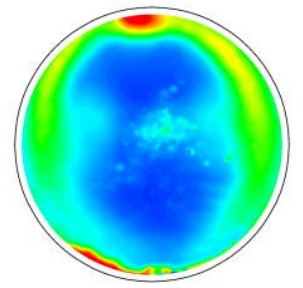

c) $\mathrm{VG} 2$

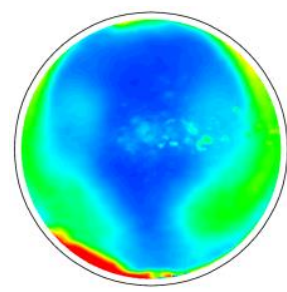

h) VG7

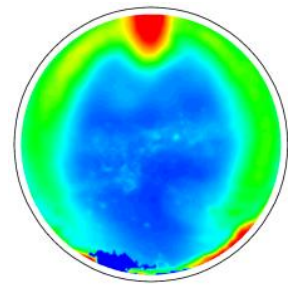

d) VG3

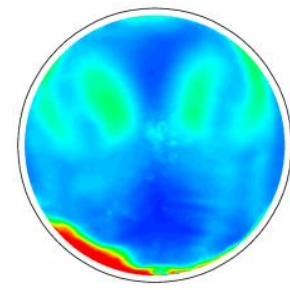

i) VG8

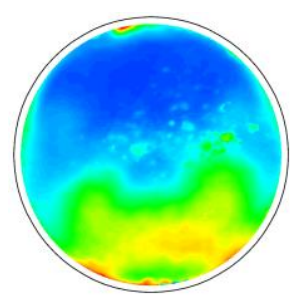

e) VG4

$$
\sigma_{\alpha}\left(^{\circ}\right)
$$

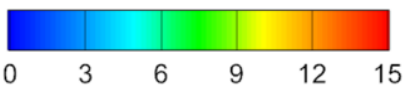

Fig. 15 Swirl angle standard deviation from S-PIV at $M_{\text {in }}=0.27$

\section{Swirl distortion analysis}

The quantification of the effect of the vortex generators upon the distorted flow field was assessed using the swirl distortion descriptors presented in Section II. The descriptors are first calculated for each ring for each snapshot. The area weighted average of the ring based descriptors is then computed to obtain a single value per snapshots. The statistics of the area-averaged descriptors are then calculated. An assessment of the swirl distortion descriptors for the datum configuration was previously done by Zachos et al. [15] . The descriptor characteristics for each vortex generator cases are presented in Table 3.

\section{Swirl intensity analysis}

As anticipated by the time-averaged swirl angle distribution, the mean swirl intensity ( $\mathrm{SI}_{\text {mean }}$ ) for the vortex generator configurations varies from one case to another (Fig. 16). VG2, VG3 and VG9 present similar or higher values of $\mathrm{SI}_{\text {mean }}$ compared to the datum flow field $\left(\mathrm{SI}_{\text {mean }}=8.66\right)$, with values of approximately $8.3^{\circ}, 9.4^{\circ}$ and $8.9^{\circ}$, respectively. However the maximum swirl intensity is reduced for these three configurations, with $S I_{\max }=10.1^{\circ}$, $11.9^{\circ}$ and $11.3^{\circ}$ respectively, compared with $15.5^{\circ}$ for the datum configuration. For VG2, VG3 and VG9, the difference between the maximum value of SI and their mean value is reduced. The ratio $S I_{\text {max }} / S I_{\text {mean }}$ for the datum configuration is 1.8 while for $\mathrm{VG} 2, \mathrm{VG} 3$ and $\mathrm{VG} 9, S I_{\text {max }} / S I_{\text {mean }}=1.2,1.3$ and 1.3 respectively. In addition, the standard deviation of SI $\left(\sigma_{S I}\right)$ is reduced from $1.61^{\circ}$ for the datum configuration to $0.65^{\circ}, 0.66^{\circ}$ and $0.65^{\circ}$ for VG2, 
VG3 and VG9 respectively. Therefore, although the new secondary flows generated can slightly increase the average level of $\mathrm{SI}_{\text {mean }}$ at the AIP, it also reduces the unsteadiness of the flow field and its peak swirl intensity values.

Table 3 Area-averaged swirl descriptors statistical properties at $M_{\text {in }}=0.27$

\begin{tabular}{ccccccccccc}
\hline & Datum & VG1 & VG2 & VG3 & VG4 & VG5 & VG6 & VG7 & VG8 & VG9 \\
\hline$S I_{\text {mean }}$ & 8.66 & 5.54 & 8.27 & 9.43 & 4.90 & 4.93 & 4.39 & 6.36 & 5.77 & 8.89 \\
$\sigma_{S I}$ & 1.61 & 0.58 & 0.65 & 0.66 & 0.47 & 0.39 & 0.32 & 0.57 & 0.44 & 0.65 \\
$S I_{\text {max }}$ & 15.51 & 8.46 & 10.13 & 11.91 & 6.86 & 6.31 & 5.27 & 8.83 & 7.59 & 11.26 \\
\hline$S D_{\text {mean }}$ & 0.00 & 0.00 & 0.00 & -0.08 & -0.08 & -0.08 & 0.03 & 0.01 & 0.04 & -0.02 \\
$\sigma_{S D}$ & 0.38 & 0.20 & 0.13 & 0.13 & 0.20 & 0.12 & 0.13 & 0.14 & 0.16 & 0.10 \\
$S D_{\text {max }}$ & 0.90 & 0.60 & 0.42 & 0.28 & 0.62 & 0.28 & 0.51 & 0.54 & 0.51 & 0.30 \\
\hline$S P_{\text {mean }}$ & 0.98 & 1.15 & 1.06 & 1.02 & 1.36 & 1.22 & 1.46 & 1.14 & 1.25 & 1.09 \\
$\sigma_{S P}$ & 0.15 & 0.13 & 0.09 & 0.08 & 0.20 & 0.14 & 0.17 & 0.12 & 0.12 & 0.09 \\
$S P_{\text {max }}$ & 1.61 & 1.73 & 1.43 & 1.29 & 2.22 & 1.90 & 2.10 & 1.61 & 1.72 & 1.40 \\
$S P_{\text {min }}$ & 0.57 & 0.68 & 0.79 & 0.79 & 0.84 & 0.91 & 1.02 & 0.82 & 0.85 & 0.84 \\
\hline
\end{tabular}

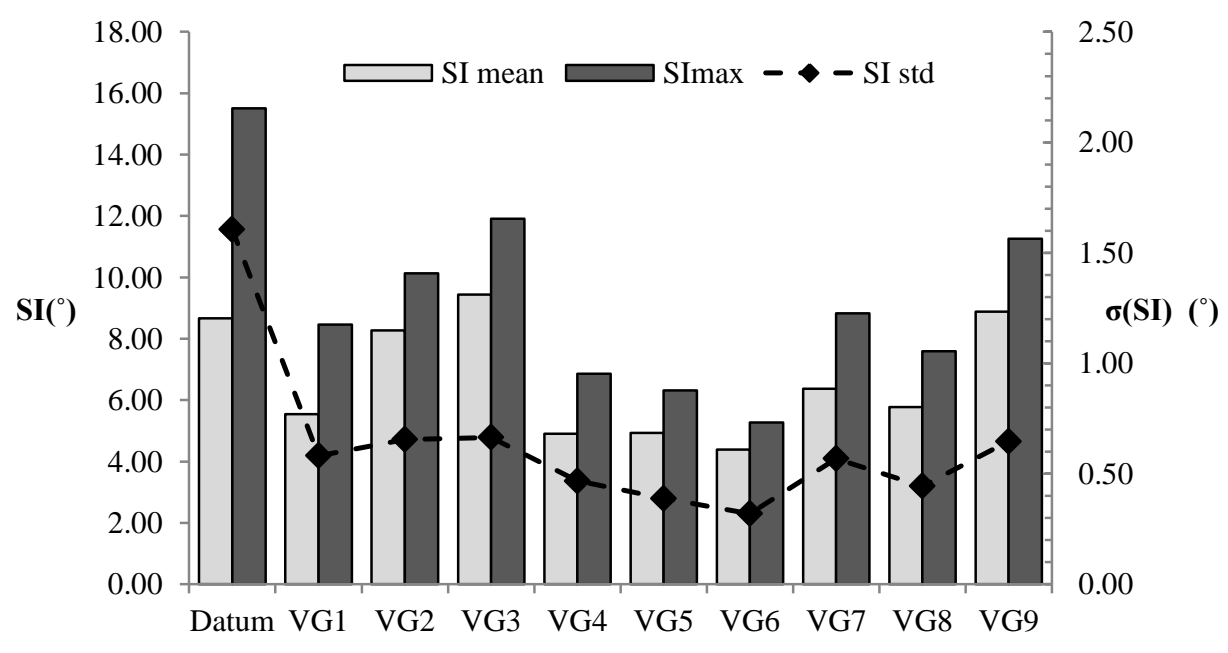

Fig. 16 Swirl intensity statistical properties at $M_{i n}=0.27$

The rest of the VG configurations present lower $\mathrm{SI}_{\text {mean }}, \mathrm{SI}_{\max }$ and $\sigma_{S I}$ value compared with the datum configuration (Fig. 16). The configuration VG4 to VG7, with the lowest height investigated $\left(h / \delta_{\text {in }}=0.63\right)$, 
performs better with a range of $\mathrm{SI}_{\text {mean }}$ between $4.4^{\circ}$ and $6.4^{\circ}$, and $\mathrm{SI}_{\max }$ between $5.3^{\circ}$ and $8.8^{\circ}$. The $\mathrm{SI}$ fluctuations are also substantially reduced compared with the datum configuration. VG6 $\left(\theta_{s}=65.5^{\circ}, h / \delta_{\text {in }}=0.63, \Delta x / \delta_{\text {in }}=20.8\right)$ present the best statistics in terms of SI, the $\sigma_{S I}$ value is reduced by $80 \%$ and the $\mathrm{SI}_{\max }$ by $66 \%$ compare with the datum configuration. Overall the average $\sigma_{S I}$ reduction over all the VG cases is about $67 \%$ compare with the datum configuration.

\section{SP-SD Cloud maps}

The analysis of the descriptors SD and SP can provide an insight of the instantaneous flow structure and its variations. The instantaneous relationship between the two descriptors can be identified with cloud maps as presented for VG1, VG2, VG4 and VG5 in Fig. 17a-d. Each point corresponds to the value of SP-SD for one single snapshot. Although the time averaged flow field for the datum configuration exhibit a symmetrical twin vortex pattern, the unsteady nature flow field was previously demonstrated by Zachos et al. [15] and is highlighted by the scatter of the SP-SD cloud map (Fig. 17a-d). The large range of SP values from 0.57 to 1.61 combined with SD values ranging from $\mathrm{SD}=-0.84$ to +0.90 (Table 3 ) for the datum configuration demonstrates the deviation of the swirl pattern from the well-known symmetrical vortex pair arrangement. Furthermore, events associated with $S \mathrm{P} \cong 0.5$ and $\mathrm{SD} \cong \pm 1$ characterise the presence of a co- or counter rotating bulk swirl at the AIP which is proven to affect the surge margin [4]. Zachos et al. [15] identified that a biased flow toward one side were generally associated with large value of $\mathrm{SI}_{\text {mean }}$. These extreme events were also confirmed by Gil-Prieto et al. [16] in which the study of the inner ring $(\mathrm{r} / \mathrm{R}=0.32)$ showed a tri-modal flow field: negative bulk swirl $(\mathrm{SP}=0.5, \mathrm{SD}=-1)$, symmetric twin-swirl ( $\mathrm{SP}=1, \mathrm{SD}=0$ ) and positive bulk swirl $(\mathrm{SP}=0.5, \mathrm{SD}=+1)$. It was concluded from the SP-SD relationship that the flow experiences a bulk-to-twin switching mechanism in which the flow oscillates from one positive or negative bulk swirl to twin vortex pattern [16].

The reduction in unsteadiness for SD and SI due to the new secondary flows generated by the effect of the vortex generators is highlighted by the cloud plot for VG1, VG2, VG4 and VG5 (Fig. 17e-f). The VGs have a significant impact on the flow field structure where the bulk swirl events associated with $\mathrm{SP} \cong 0.5$ and $\mathrm{SD} \cong \pm 1$ are suppressed. Among all the VG configurations, VG1 $\left(\theta_{s}=67.5^{\circ}, h / \delta=0.88, \Delta_{x} / \delta=4.63\right)$ presents events that are the closest to a bulk swirl pattern with $\mathrm{SP}_{\min }$ and $\left|\mathrm{SD}_{\max }\right|$ values respectively 0.68 and 0.60 (Table 3 ). However this corresponds to the presence in the section of an asymmetric vortex pair with one covering a greater portion of the 
AIP while the other is confined to a smaller area. Therefore the flow topology of extreme events for the VG cases moves away from a bulk swirl topology toward a relatively less unsteady system of multiple regions of swirling flow as $\mathrm{SP}_{\text {mean }}$ is higher than unity for all the configurations (Table 3). The range of SD value for VG2 and VG5 is reduced to [-0.45-0.45] associated with a one third reduction of $\sigma_{S D}$ compared to the datum configuration. Compared to the other VG cases, a higher range of $\mathrm{SD}$ values for VG4 is comprised between $\mathrm{SD}=[-0.65,0.65]$ due to the higher swirl unsteadiness on the bottom half of the AIP (Fig. 15). Furthermore, SP values for VG4 typically range from 0.8 to 2.0 which indicate a relatively unstable system of multiple swirling structures at the AIP. Therefore, the relationship between SD and SP demonstrates the suppression of the bulk-to-twin switching mechanism due to the vortex generators. Instead, a system of multiple regions of swirling flow induced by the new secondary flows is generated at the AIP.

\section{SD-SI Cloud maps}

For the datum configuration, high SI values are generally associated with large excursions of SD value from the mean (Fig. 17e-f) as previously highlighted by Zachos et al. [15]. The investigation by Gil-Prieto et al. [16] for the inner ring $(r / R=0.32)$ of the AIP also concluded that the highest SI values were promoted by bulk swirl events with SD values of $\mp 1$ and $\mathrm{SP}=0.5$. It is important to notice that the present investigation is calculating the distortion descriptors over the full AIP and therefore local extreme events in the AIP have a tendency to be damped by the area averaged process over the rings. This explains why nominally pure bulk swirl is not demonstrated by the SD-SP cloud maps (Fig. 17).

Nevertheless, those extreme events are still visible for the datum configuration and a significant effect of the VGs on the flow fields is visible at the AIP. The use of VGs reduces the SI-SD scatter for all the configurations. This was anticipated by the great reduction in standard deviation of both SI and SD (Table 3) for the VG configurations. The maximum values of SI for the VG cases are no longer associated with large excursions from the mean flow field. More importantly, large excursion in SI from the mean value is not recurrent as it was the case for the datum configuration. Therefore not only do the VGs reduce the $\mathrm{SI}_{\text {mean }}$, but they also keep the fluctuation relatively small for all the range of SD. 

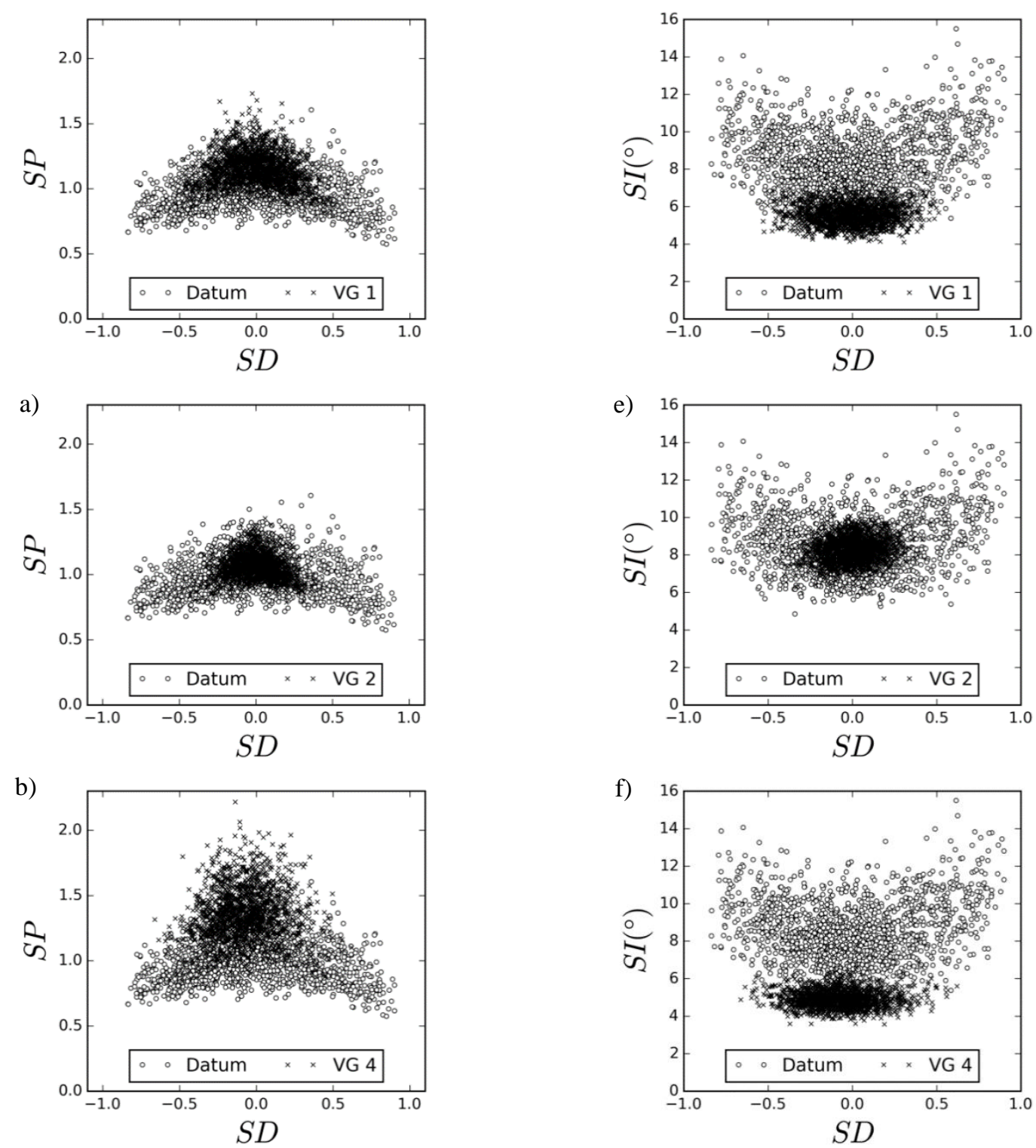

c)

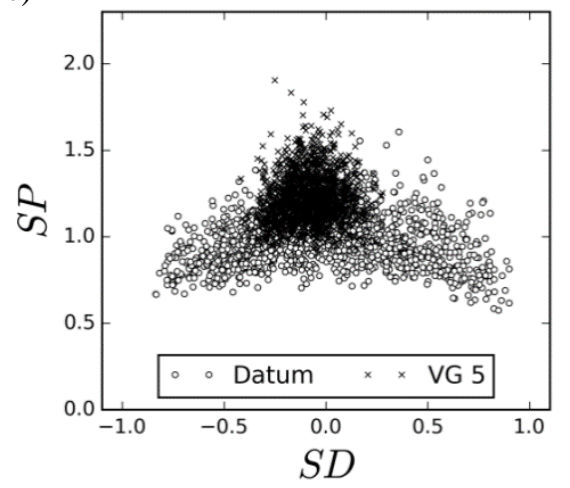

d)

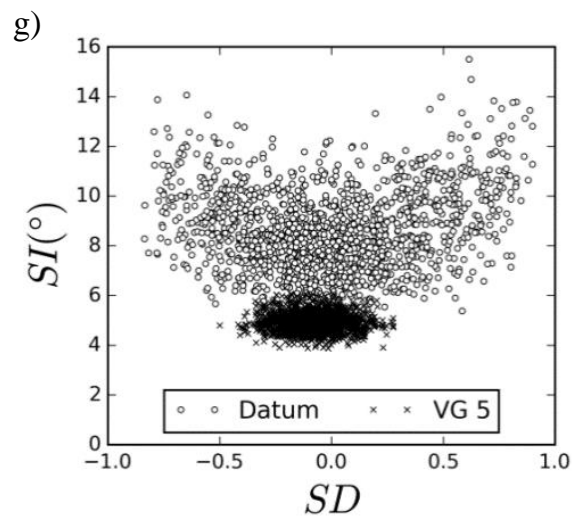

h)

Fig. 17 SP-SD (a-d) and SI-SD (e-f) distortion cloud plots at $M_{i n}=0.27$ 
The instantaneous flow field at the AIP for VG5 $\left(\theta_{s}=97.5^{\circ}, h / \delta=0.63, \Delta x / \delta_{\text {in }}=14\right)$, which presents the best compromise between the improvements in the SI, SP and SD characteristics, is also qualitatively assessed. As indicated by the standard deviation of the swirl angle distributions (Fig. 15f), the swirl regions generated by the new secondary flows on each side of the AIP does not merged together and stay confined in the periphery on the section (Fig. 19). Sample plots of instantaneous in-plane velocity stream lines reveal a multiple swirl patterns generated on both side of the AIP. It can be pointed out that the S-PIV system successfully captured both large and small scale structures at the AIP which is not possible to obtain with conventional total pressure measurements from an $8 \times 5$ rake.

Table 4 Desciptors statistics for the datum configuration and VG case 5

\begin{tabular}{|c|c|c|c|c|c|c|}
\hline & \multicolumn{3}{|c|}{ Datum at $\mathrm{Min}=0.27$} & \multicolumn{3}{|c|}{ VG 5} \\
\hline & SI & SP & SD & SI & SP & SD \\
\hline mean & $8.7^{\circ}$ & 0.98 & 0.00 & $4.9^{\circ}$ & 1.22 & -0.08 \\
\hline$\sigma$ & $1.6^{\circ}$ & 0.15 & 0.38 & $0.4^{\circ}$ & 0.14 & 0.12 \\
\hline Skewness & 0.6 & 0.44 & 0.15 & 0.3 & 0.65 & 0.02 \\
\hline kurtosis & 3.2 & 3.30 & 2.40 & 3.1 & 3.69 & 2.82 \\
\hline $\operatorname{Max}$ & $15.5^{\circ}$ & 1.61 & 0.90 & $6.3^{\circ}$ & 1.90 & 0.28 \\
\hline Min & $4.9^{\circ}$ & 0.57 & -0.84 & $3.9^{\circ}$ & 0.91 & -0.40 \\
\hline
\end{tabular}

The skewness and kurtosis for the datum configuration and VG5 are added to the statistical assessment of the swirl descriptors (Table 4). In both cases, the SI descriptor shows a distribution close to normal with a kurtosis equal to 3.2 and 3.1 for the datum configuration and VG5 respectively. However the standard deviation of SI for VG5 is decreased by $75 \%$ compared with the datum configuration. The reduced scatter in SI value is further indicated by peak-to-peak values which are $2.4^{\circ}$ and $10.6^{\circ}$ for VG5 and the datum configuration respectively (Table 4). Both configurations have a positive skewness indicating the greater likelihood of the occurrence of adverse swirl distortion events. However the skewness of SI for VG5 is half the one of the datum configuration. The mean value of SP for the datum configuration and VG5 are 0.98 and 1.22 respectively. As a result the likelihood to obtain a bulk swirl event $(\mathrm{SP}=0.5, \mathrm{SD}= \pm 1)$ is substantially lower for VG5. On the other end, multiple pairs of vortices exist in the unsteady flow field for VG5. A greater likelihood of occurrence of multiple swirling structures at the AIP is characterized by a greater SP skewness of 0.65 for VG5. The kurtosis of SD for the datum configuration is 2.4 , 
lower than the normal distribution value of 3.0. As a result a flatter distribution is achieved for SD which indicates the higher probability of bulk swirl event at the AIP with SD value from -0.84 to 0.90 . In contrast for VG5 the SD ranges from -0.28 to 0.40 which confirms the suppression of bulk swirl events at the AIP (Table 4). Overall VG5 presents a system of multiple pair of vortices with a reduced likelihood of extreme swirl events compared with the datum configuration.
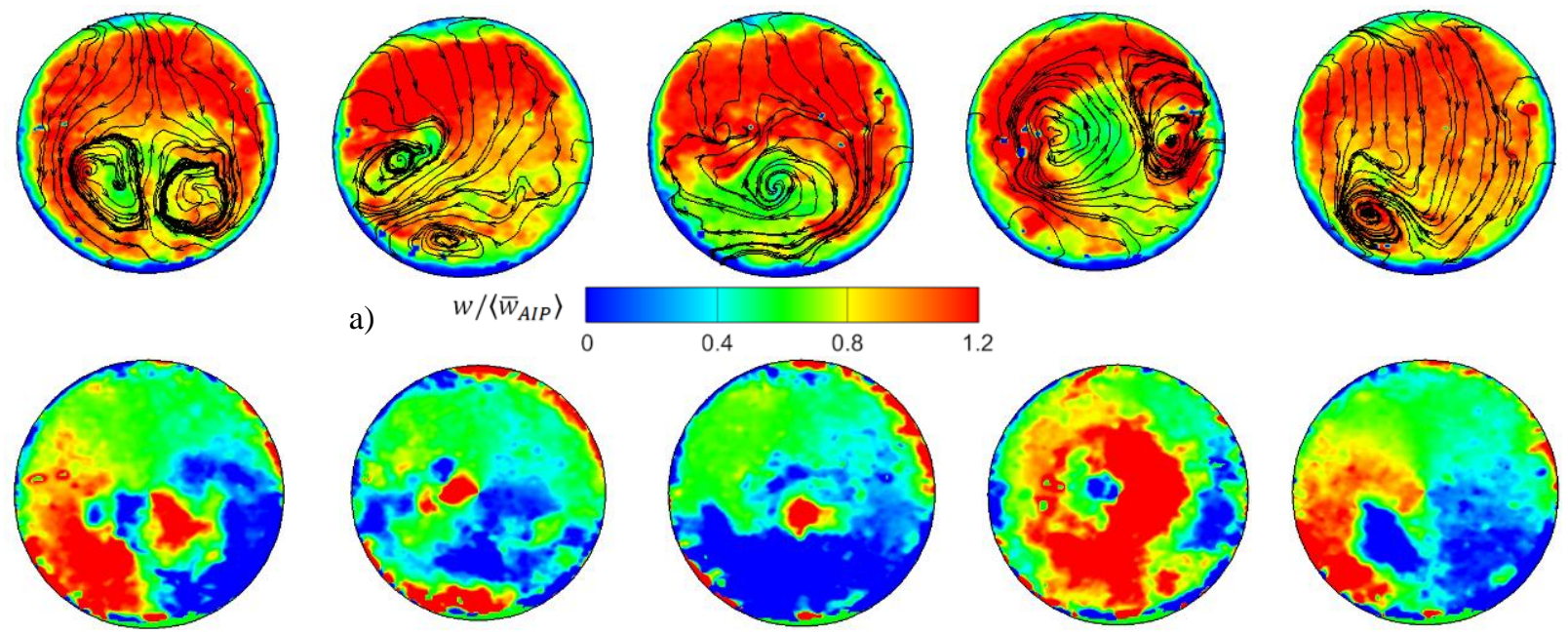

a)

$w /\left\langle\bar{w}_{A I P}\right\rangle$

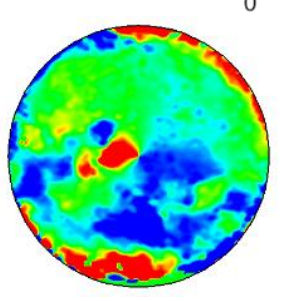

b)

$$
\alpha\left(^{\circ}\right)
$$
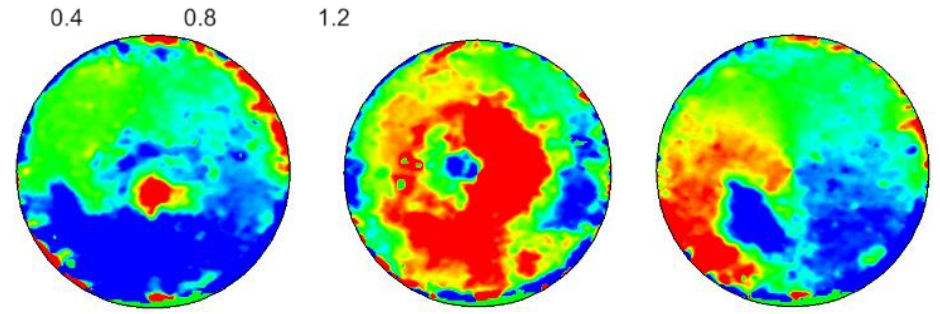

Fig. 18 Datum configuration instantaneous out of plane velocity (a) and swirl angle (b) flow field from S-PIV at $M_{i n}=0.27$
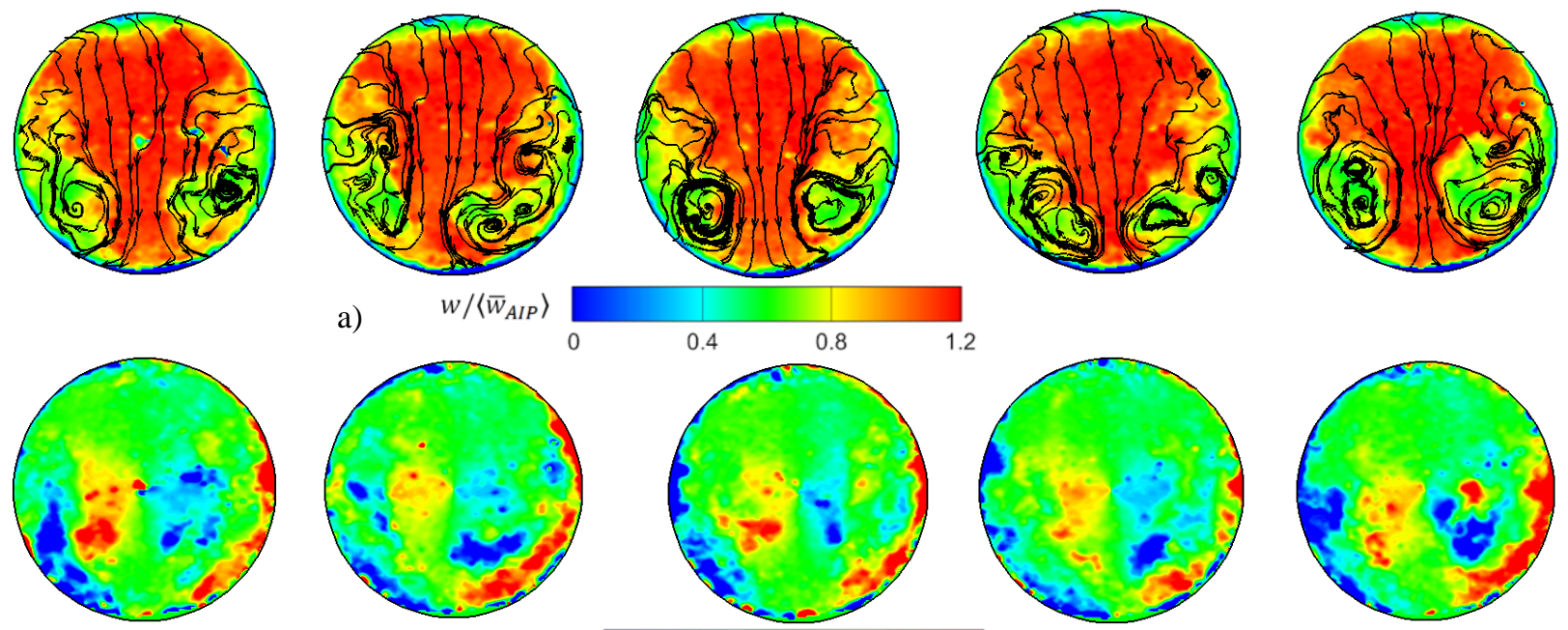

a)

$w /\left\langle\bar{w}_{A I P}\right\rangle$

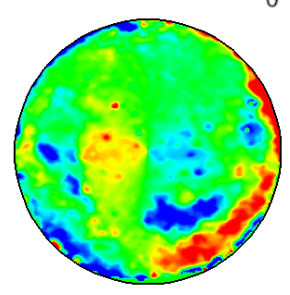

$\alpha\left(^{\circ}\right)$
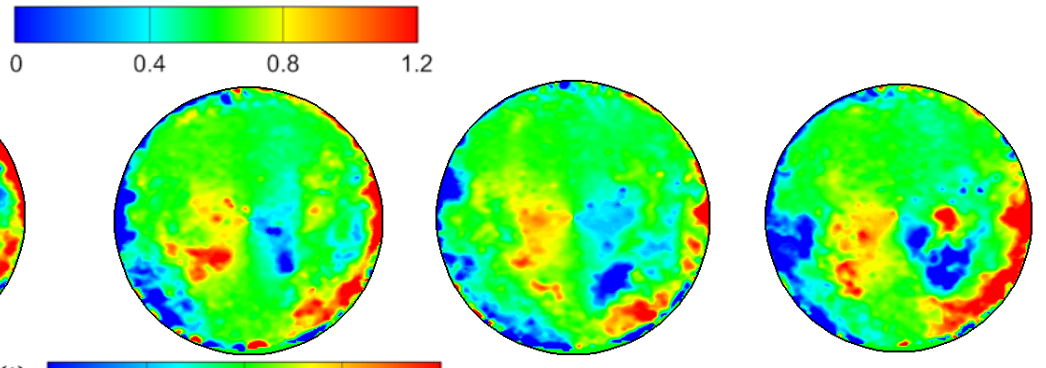

b)

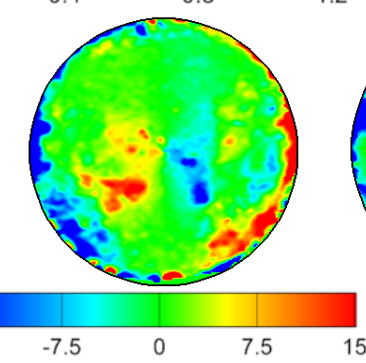

Fig. 19 VG5 configuration instantaneous out of plane velocity (a) and swirl angle (b) flow field from S-PIV at

$\mathrm{M}_{\mathrm{in}}=\mathbf{0 . 2 7}$ 


\section{Design parameter evaluation}

Both total pressure measurements and S-PIV measurements have shown a variation in total pressure recovery and distortion descriptors due to a change in the vortex generator configuration. Three VG design variables were mainly investigated for this survey corresponding to the circumferential extent ( $\left.\theta_{s}\right)$ the VGs height $\left(h / \delta_{\text {in }}\right)$ and their centerline axial location $\left(\Delta x / \delta_{\text {in }}\right)$. Furthermore, the number of VGs and lateral spacing $\left(\alpha_{s}\right)$ were also varied at a constant $\theta_{s}$. Although the survey was not seen as an optimisation process, some conclusions can be made on the isolated effect of those design variables.

The effect of $\theta_{s}$, defined as the circumferential location where the vortex generator arrays are placed for each half of the duct (Fig. 3), was assessed with VG1, VG2 and VG3 with $\theta_{\mathrm{s}}=67.5^{\circ}, 97.5^{\circ}$ and $157.5^{\circ}$ respectively. The circumferential spacing between the vortex generators was kept constant, which resulted in 10, 14 and 22 vortex generators for VG1, VG2 and VG3, respectively. For these configurations, the mean flow presents a pair of counterrotating vortices (Fig. 12). The core location of those vortices are circumferentially moved towards the upper sector of AIP as the circumferential extent $\left(\theta_{s}\right)$ is increased. However the assessment of the unsteady snapshot provided by the synchronous S-PIV measurements revealed that multiple pairs of vortices were present at the AIP. This is confirmed by the $\mathrm{SP}_{\text {mean }}>1$ for each of these configurations (Table 3). When $\theta_{s}$ varies from $67.5^{\circ}$ to $157.5^{\circ}, \mathrm{SP}_{\text {mean }}$ is reduced from 1.15 to 1.02 and the minimum to maximum range decreased from 1.05 to 0.50 . Therefore the flow structure tends toward a single pair of vortices as $\theta_{s}$ increases (Fig. 20a). However, the addition of the vortex generators also generates stronger new secondary flows characterized by an increase in absolute swirl angle with region of $|\alpha|=35^{\circ}$ near the periphery of the AIP (Fig. 13c, d). The $\mathrm{SI}_{\text {mean }}$ is also increased from $5.5^{\circ}$ to $9.4^{\circ}$ (Fig. 20b). Furthermore, with a high $\theta_{s}$, the potency of the VG induced secondary flow results in a convergence of the swirling structure at the top of the AIP and a region of low streamwise velocity with an increase in the swirl unsteadiness in the upper sector.

VG4, VG2 and VG9 present the same design characteristics $\left(\theta_{s}=97.5^{\circ}, \Delta \mathrm{x} / \delta_{i n}=4.63\right)$ except for the VG heights $\left(\mathrm{h} / \delta_{\text {in }}\right)$ which are respectively $0.63 \delta_{\text {in }}, 0.88 \delta_{\text {in }}$ and $1.25 \delta_{\text {in. }}$. For an inlet Mach number of 0.60 , the DC60 varies monotonically from 0.10 to 0.54 as the VGs height increases from $0.63 \delta_{\text {in }}$ to $1.25 \delta_{\text {in }}$ (Fig. 11). The associated PR values are relatively unchanged. The level of swirl intensity at the AIP is also increased by the VGs height from $4.9^{\circ}$ to $8.9^{\circ}$ for a variation in $\mathrm{h} / \delta_{\text {in }}$ from 0.63 to 1.25 respectively (Fig. 21a). The increase of the swirl intensity fluctuation due to the VGs height can be explained by the larger mean value achieved. Due to the higher VGs, the 
flow topology for VG2 and VG9 does not present any swirl angle fluctuations at the bottom of the AIP as it was the case for VG4 (Fig. 15e). However the higher VGs (VG2 and, VG9) promote fluctuations on the top of the AIP. The mean out of plane velocity field (Fig. 12j) suggests large and strong swirling structures due to the highest VG configuration (VG9) which also generates swirl angle fluctuations in the usually-steady core of the AIP (Fig. 15j). An increase in $\mathrm{h} / \delta_{\text {in }}$ tends to stabilize the flow structure with $\mathrm{SP}_{\text {mean }}$ values converging toward unity (Fig. $21 \mathrm{~b}$ ). Overall the increase in $\mathrm{h} / \delta_{\text {in }}$ generates stronger secondary flows with higher values of $\mathrm{SI}_{\text {mean }}$, however it also stabilizes the flow by reducing the swirl unsteadiness.
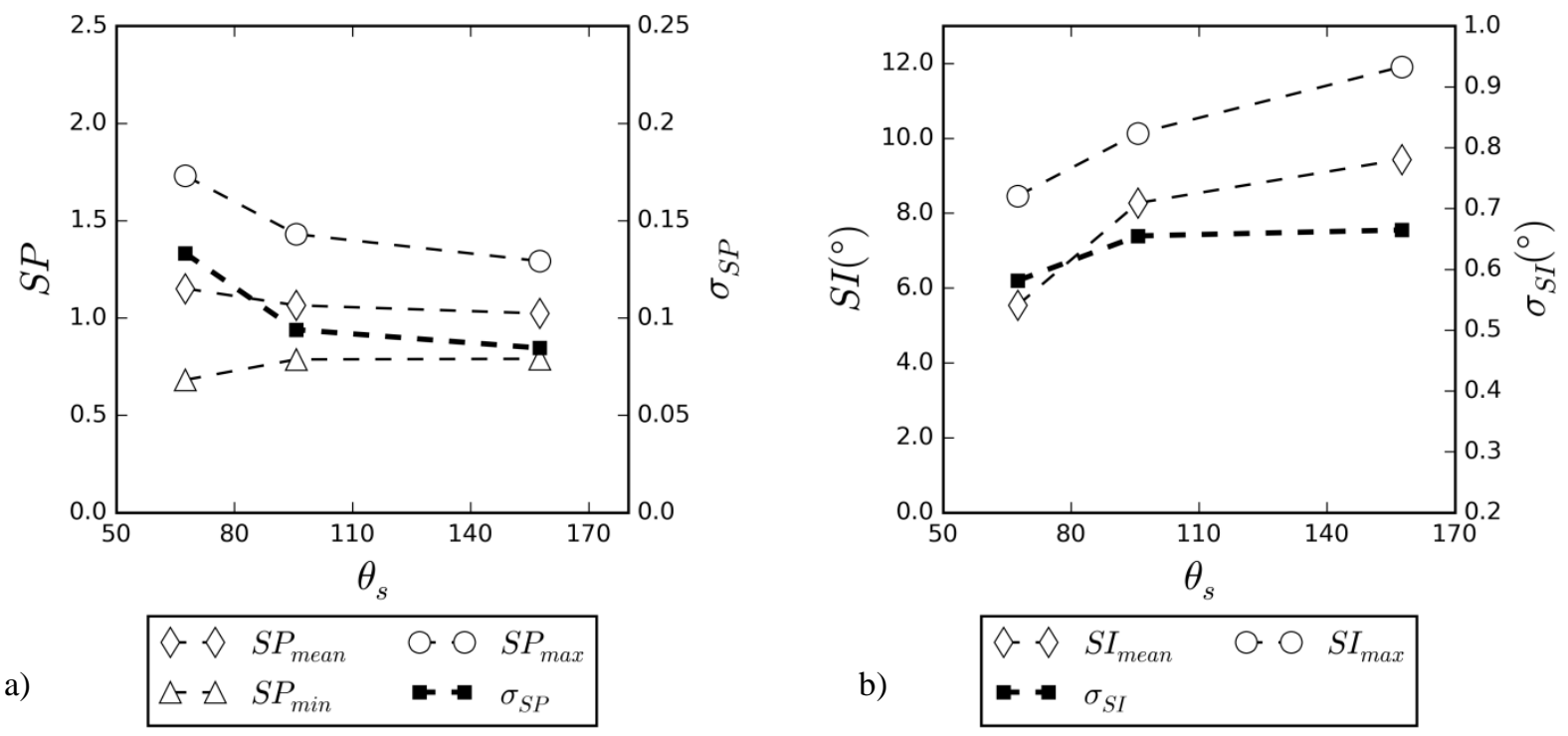

\section{Fig. 20 Effect of $\theta_{s}$ on SP (a) and SI (b) statistics}

VG4 and VG5 are used in order to assess the effect of the axial position $\left(\Delta x / \delta_{\text {in }}\right)$ of the VGs relative to the flow separation onset point. The two configurations have the same VG height of $0.63 \delta_{\text {in }}$ and a circumferential extent of $97.5^{\circ}$. There are notable differences in the mean flow topology between VG4 and VG5. VG4 is located at $\Delta \mathrm{x} / \delta_{\text {in }}$ of 4.63 and results in two pairs of weak vortices close to the periphery of the AIP. VG5, which is positioned further upstream at $\Delta \mathrm{x} / \delta_{\mathrm{in}}=14$ shows a main single pair of vortices slightly off the surface of the duct (Fig. 12f). The pressure distortion descriptor DC60 increases as the VGs are placed further upstream from the nominal centerline separation point (Fig. 11). However the duct efficiency for VG5 improves, with a PR $=0.970$ compared with $\mathrm{PR}=0.966$ for $\mathrm{VG} 4$. The increase in $\Delta \mathrm{x} / \delta_{\text {in }}$ from 4.63 to 14 reduces the maximum levels of swirl intensity by $8.0 \%$ and decreases the SI standard deviation by $17 \%$. The flow at the AIP is also stabilized with a reduction of $\sigma_{S P}$ from 0.20 to 0.12 when $\Delta x / \delta_{\text {in }}$ varies from 4.63 to 14 . Therefore, overall, a better performance is achieved with the 
VGs located at a distance $\Delta x / \delta_{\text {in }}=14$ from the nominal centreline separation point. VG4 $\left(\Delta x / \delta_{\text {in }}=4.63\right)$ could simply be placed too close to the separation point. The relative height of VG4 $\left(0.63 \delta_{\text {in }}\right)$ to the local boundary layer thickness could be too small to fully guide the flow and control the secondary flows as opposed to $\mathrm{VG} 2\left(\Delta x / \delta_{\text {in }}=\right.$ $4.63, \mathrm{~h} / \delta_{\text {in }}=0.88$ ) which successfully reduces the swirl unsteadiness at the bottom of the AIP.
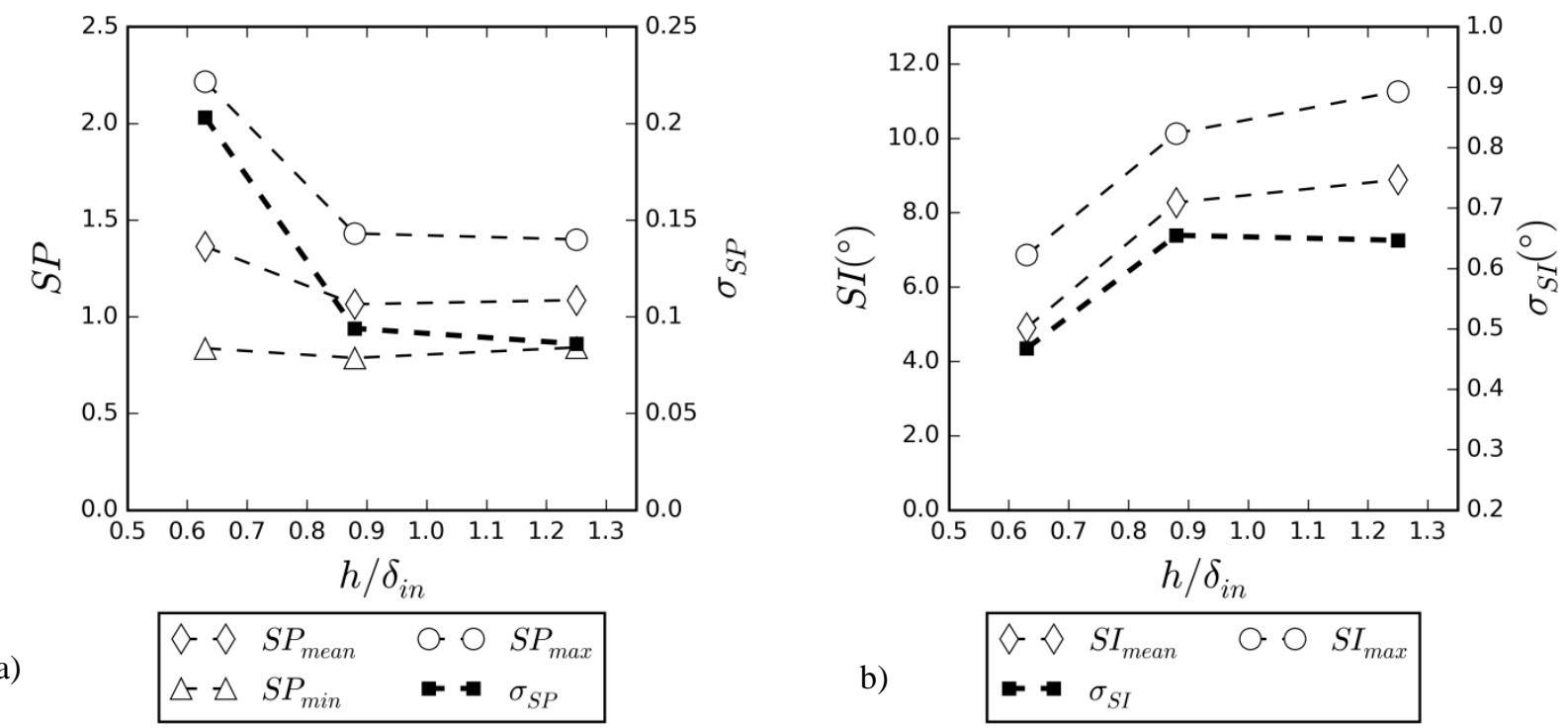

Fig. 21 Effect of $h / \delta_{\text {in }}$ on SP statistics SP (a) and SI (b) statistics

\section{Conclusion}

This paper presents the effect of passive flow control devices on the unsteady flow field at the exit of a convoluted S-duct intake. The detailed characteristics of the datum and controlled flow fields at the AIP have been assessed through the use of high resolution and synchronous S-PIV measurements. This enabled the statistical evaluation of the swirl distortion descriptors at the AIP to quantify the effect of vortex generators (VGs) on the unsteady distorted flow field. In addition, the total pressure flow field at the AIP was measured to determine the pressure based distortion descriptors. Thus, for the first time total pressure and 3-D velocity based measurements could be conjointly assessed at the exit of an S-duct.

The total pressure deficit typically observed at the AIP is associated with a pair of counter rotating vortices. Overall, the addition of vortex generators changes the flow separation and secondary flows and tends to relocate the main loss and vortical regions towards the periphery of the AIP. As a result, the DC60 is substantially affected with a reduction of up to $50 \%$ compared with the datum configuration. However, the addition of VGs can both increase 
and reduce the DC60 relative to the datum case. Pressure recovery (PR) was improved in all the VG cases with a reduction of $30 \%$ in pressure loss. The VGs can also generate substantially higher levels of swirl intensity at the AIP compared with the datum configuration. In these cases, large swirl angles up to $35^{\circ}$ are measured near the periphery of the AIP. However $\mathrm{SI}_{\text {mean }}$ was decreased in most of the VG cases, with a reduction of nearly $50 \%$ for some configuration.

The synchronous S-PIV measurements revealed the unsteady nature of the flow field at the AIP. For the datum configuration, large deviations from a pair of twin vortex were observed to be associated with a high value of SI up to nearly twice the $\mathrm{SI}_{\text {mean }}$. Those extreme events are identified by the instantaneous swirl descriptors SP and SD that demonstrated the presence of bulk swirl events at the AIP. As a result, large fluctuations in swirl angle are generated at the AIP. The instantaneous flow field for the VG cases also demonstrated unsteady features with typically multiple pairs of vortices present at the AIP. However, unlike the datum configuration, the positions of these vortices are confined to the periphery of the AIP. As a result, all the VG configurations contributed to the reduction of the flow unsteadiness by reducing the swirl angle fluctuations with an overall $67 \%$ reduction in $\sigma_{S I}$ compare with the datum configuration. The bulk-to-twin swirl switching mechanism identified for the datum configuration is suppressed by the VGs. Consequently, bulk swirl extreme events identified for the datum configuration are suppressed which substantially reduce the overall $\mathrm{SI}_{\max }$ by $45 \%$ for the VG configurations.

The studies also demonstrated the sensitivity of the flow field at the AIP regarding key VG design parameters. Overall it was found that an increase in circumferential spacing $\theta_{s}$ tends to stabilize the flow. A relatively large value of $\theta_{s}\left(97.5^{\circ}\right.$ to $\left.157.5^{\circ}\right)$ circumferentially move the location of the vortices towards the periphery of the upper sector of the AIP and increase the swirl intensity. However, high values of $\theta_{s}$ lead to the promotion of the unsteadiness in the upper sector with substantial losses generated in both total pressure and streamwise velocity. The flow pattern was also very sensitive to the VG height. Lower VG heights, relative to the boundary layer thickness, tend to generate less swirl intensity and a better PR. However they have a reduced effect on the classical secondary flows and consequently more swirl unsteadiness at the AIP.

\section{References}

[1] Liebeck, R. H., "Design of the Blended Wing Body Subsonic Transport," AIAA Journal of Aircraft, Vol. 41, No. 1, 2004, pp. $10-25$.

Doi: 10.2514/1.9084 
[2] Vakili, A., Wu, J., Liver, P., and Bhat, M., "Measurements of compressible secondary flow in a circular S-duct," 16th Fluid and Plasmadynamics Conference, AIAA 83-1739, Denvers, MA, 1983.

Doi: $10.2514 / 6.1983-1739$

[3] Wellborn, S. R., Reichert, B. A., and Okiishi, T. H., "Study of the Compressible Flow in a Diffusing S-Duct," AIAA Journal of Propulsion and Power, Vol. 10, No. 5, 1994, pp. 668-675.

Doi: $10.2514 / 3.23778$

[4] Society of Automotive Engineers, “A Methodology for Assessing Inlet Swirl Distortion,” Aerospace Information Report 5686, Warrendale, PA, 2007.

[5] Society of Automotive Engineers, "Inlet Total Pressure Distortion Considerations for Gas Turbine Engines," Aerospace Information Report 1419a, Warrendale, PA, 1999.

[6] Cousins, W., T., "History, Philosophy, Physics and Future Directions of Aircraft Propulsion System / Inlet Integration," Proceedings of ASME Turbo Expo 2004, GT2004-54210, Vienna, Austria, 2004.

[7] Rademakers, R. P. M., Bindl, S., and Niehuis, R., "Effects of Flow Distortions as They Occur in S-Duct Inlets on the Performance and Stability of a Jet Engine," ASME Journal of Engineering for Gas Turbines and Power, Vol. 138, No. 2, 2015, p. 022605 .

Doi: $10.1115 / 1.4031305$

[8] Wenzel, L. M., and Blaha, R. J., “Analysis of dynamic inlet distortion applied to a parallel compressor model,” NASA TM3522, May 1977.

[9] Stevens, C. H., Spong, E. D., and Hammock, M. S., "F-15 inlet/engine test techniques and distortion methodologies studies. Volume 1: Technical discussion,” NASA CR-144866, June 1978.

[10] Bowditch, D. N., and Coltrin, R. E., “A Survey of Inlet/Engine Distortion Compatibility,” NASA TM-83421, June 1983.

[11] Reichert, B. A., and Wendt, B. J., "Improving curved subsonic diffuser performance with vortex generators,"AIAA Journal, Vol. 34, No.1 , 1996, pp. 65-72.

Doi: $10.2514 / 3.13022$

[12] Garnier, E., Leplat, M., Monnier, J. C., and Delva, J., "Flow control by pulsed jet in a highly bended S-duct," 6th AIAA Flow Control Conference, AIAA 2012-3250, 2012, New Orleans, Louisiana, USA, 2012.

Doi: 10.2514/6.2012-3250

[13] Berens, T. M., Delot, A. L., Chevalier, M., and Van Muijden, J., "Numerical simulations for high offset intake diffuser flows," 52 ${ }^{\text {nd }}$ AIAA Aerospace Sciences Meeting, AIAA 2014-0371, National Harbor, Maryland, USA, 2014.

Doi: 10.2514/6.2014-0371

[14] Garnier, E., "Flow Control by Pulsed Jet in a Curved S-Duct: A Spectral Analysis," AIAA Journal, Vol. 53, No. 10, 2015, 
pp. 2813-2827.

Doi: $10.2514 / 1 . J 053422$

[15] Zachos, P. K., MacManus, D. G., Gil-Prieto, D., and Chiereghin, N., "Flow Distortion Measurements in Convoluted Aero Engine Intakes," AIAA Journal (accepted for publication, In Press), 2016.

[16] Gil-Prieto, D., MacManus, D. G., Zachos, P. K., and Tanguy, G., “Convoluted Intake Distortion Measurements Using Stereo Particle Image Velocimetry,” $34^{\text {th }}$ AIAA Applied Aerodynamics Conference, ID 2426083, Washington, D.C, 2016.

[17] Sullerey, R. K., Mishra, S., and Pradeep, A. M., “Application of boundary layer fences and vortex generators in improving performance of S-duct diffusers," Journal of Fluids Engineering, Transactions of the ASME, Vol. 124, No. 1, 2002 , pp. $136-142$. Doi: $10.1115 / 1.1436096$

[18] Ng, Y. T., Luo, S. C., Lim, T. T., and Ho, Q. W., "Three Techniques to Control Flow Separation in an S-Shaped Duct," AIAA Journal, Vol. 49, No 9, 2011, pp. 1825-1832.

Doi: 10.2514/1.J050135

[19] Paul, A. R., Ranjan, P., Patel, V. K., and Jain, A., “Comparative studies on flow control in rectangular S-duct diffuser using submerged-vortex generators," Aerospace Science and Technology, Vol. 28, No 1. 2013, pp. 332-343.

Doi: 10.1016/j.ast.2012.11.014

[20] Vakili, A., Wu, J., Liver, P., and Bhat, M., "Flow control in a diffusing S-Duct," Shear Flow Control Conference, AIAA 850524, Reston, Viriginam, 1985.

Doi: $10.2514 / 6.1985-524$

[21] Anderson, B. H., and Gibb, J., "Study on vortex generator flow control for the management of inlet distortion," AIAA Journal of Propulsion and Power, Vol. 9, No. 3, 1993, pp. 422-430.

Doi: $10.2514 / 3.23638$

[22] Anderson, B. H., and Gibb, J., "Vortex-generator installation studies on steady-state and dynamic distortion," AIAA Journal of Aircraft, Vol. 35, No. 4, 1998, pp. 513-520.

Doi. $10.2514 / 2.2340$

[23] Allan, B. G., Owens, L. R., and Lin, J. C., "Optimal design of passive flow control for a boundary-layer-ingesting offset inlet using design-of-experiments," 44th AIAA Aerospace Sciences Meeting, AIAA 2006-1049, Reno, Nevada, 2006.

Doi: $20.2514 / 6.2006-1049$

[24] Owens, L. R., Allan, B. G., and Gorton, S. A., "Boundary-Layer-Ingesting Inlet Flow Control," AIAA Journal of Aircraft, Vol. 45, No. 4, 2008, pp. 1431-1440.

Doi: $10.2514 / 1.36989$

[25] Gissen, A. N., Vukasinovic, B., McMillan, M. L., and Glezer, A., “Distortion Management in a Boundary Layer Ingestion 
Inlet Diffuser Using Hybrid Flow Control," AIAA Journal of Propulsion and Power, Vol. 30, No. 3, 2014, pp. 834-844.

Doi: $10.2514 / 1 . B 34981$

[26] Gissen, A. N., Vukasinovic, B., and Glezer, A., "Dynamics of flow control in an emulated boundary layer-ingesting offset diffuser," Experiments in Fluids, Vol. 55, No. 8, 2014, p. 1794.

Doi: 10.1007/s00348-014-1794-5

[27] McMillan, M. L., Mackie, S. A., Gissen, A. N., Vukasinovic, B., Lakebrink, M. T., Glezer, A., Mani, M., and Mace L, J., “Inlet Flow Control and Prediction Technologies for Embedded Propulsion Systems,” NASA CR-2011-217237, Dec. 2011.

[28] Yi, J., Kim, C., and Lee, B. J., “Adjoint-based design optimization of vortex generator in an s-shaped subsonic inlet,” AIAA Journal, Vol. 50, No 11, 2012, pp. 2492-2507.

Doi: $10.2514 / 1 . J 051687$

[29] Jirásek, A., "Design of vortex generator flow control in inlets," AIAA Journal of Aircraft, Vol. 43, No. 6, 2006, pp. 18861892.

Doi: $10.2514 / 1.21364$

[30] Jirásek, A., "Development and application of design strategy for design of vortex generator flow control in inlets," 44th AIAA Aerospace Sciences Meeting, AIAA 2006-1050, Reno, Nevada, 2006.

Doi: $10.2514 / 6.2006-1050$

[31] Johansson, M., "Propulsion Integration in an UAV," 24th AIAA Applied Aerodynamics Conference, AIAA 2006-2834, San Francisco, California, 2006.

Doi:10.2514/6.2006-2834

[32] Delot, A.-L., Garnier, E., and Pagan, D., "Flow control in a high-offset subsonic air intake," 47th AIAA/ASME/SAE/ASEE Joint Propulsion Conference and Exhibit, AIAA 2011-5569, San Diego, California, USA, 2011.

Doi: $10.2514 / 6.2011-5569$

[33] Delot, A.-L., and Scharnhorst, R., “Computational and Experimental Results for Flows in a Diffusing S-Duct without and with Flow Control Devices," 51st AIAA/SAE/ASEE Joint Propulsion Conference and Exhibit, AIAA-2015-3964, Orlando, FL, 2015.

Doi:10.2514/6.2015-3964

[34] Raffel, M., Willert, C., Wereley, S., and Kompenhans, J., Particle Image Velocimetry: a practical guide, $2^{\text {nd }}$ ed., SpringerVerlag, Berlin, 2007.

Doi: $10.1007 / 978-3-540-72308-0$

[35] Bissinger, N. N. and Breuer, T., Basic Principles - Gas Turbine Compatibility - Intake Aerodynamics Aspects, Encyclopaedia of Aerospace Engineering, John Wiley \& Sons, Ltd., 2010, pp. 1-10. 
Doi: 10.1002/9780470686652.eae48 
2017-05-08

Passive flow control study in an S-duct intake using Stereo Particle Image Velocimetry

Tanguy, Geoffrey

American Institute of Aeronautics and Astronautics

Geoffrey Tanguy, David G. MacManus, Pavlos Zachos, Daniel Gil-Prieto and Eric Garnier.

Passive flow control study in an S-duct intake using Stereo Particle Image Velocimetry. AIAA

Journal, Vol. 55, No. 6 (2017), pp1862-1877

http://dx.doi.org/10.2514/1.J055354

Downloaded from Cranfield Library Services E-Repository 